\title{
A finite element implementation of the isotropic exponentiated Hencky-logarithmic model and simulation of the eversion of elastic tubes
}

\author{
Boumediene Nedjar, Herbert Baaser, $^{2}$ Robert J. Martin ${ }^{3}$ and Patrizio Neff ${ }^{4}$
}

April 26, 2018

\begin{abstract}
We investigate a finite element formulation of the exponentiated Hencky-logarithmic model whose strain energy function is given by$$
W_{\mathrm{eH}}(\boldsymbol{F})=\frac{\mu}{k} e^{k\left\|\operatorname{dev}_{n} \log \boldsymbol{U}\right\|^{2}}+\frac{\kappa}{2 \hat{k}} e^{\hat{k}[\operatorname{tr}(\log \boldsymbol{U})]^{2}},
$$

where $\mu>0$ is the (infinitesimal) shear modulus, $\kappa>0$ is the (infinitesimal) bulk modulus, $k$ and $\hat{k}$ are additional dimensionless material parameters, $\boldsymbol{U}=\sqrt{\boldsymbol{F}^{T} \boldsymbol{F}}$ and $\boldsymbol{V}=\sqrt{\boldsymbol{F} \boldsymbol{F}^{T}}$ are the right and left stretch tensor corresponding to the deformation gradient $\boldsymbol{F}$, log denotes the principal matrix logarithm on the set of positive definite symmetric matrices, $\operatorname{dev}_{n} \boldsymbol{X}=\boldsymbol{X}-\frac{\operatorname{tr} \boldsymbol{X}}{n} \mathbf{1}$ and $\|\boldsymbol{X}\|=\sqrt{\operatorname{tr} \boldsymbol{X}^{T} \boldsymbol{X}}$ are the deviatoric part and the Frobenius matrix norm of an $n \times n$-matrix $\boldsymbol{X}$, respectively, and tr denotes the trace operator.

To do so, the equivalent different forms of the constitutive equation are recast in terms of the principal logarithmic stretches by use of the spectral decomposition together with the undergoing properties. We show the capability of our approach with a number of relevant examples, including the challenging "eversion of elastic tubes" problem.
\end{abstract}

Key words: exponentiated Hencky-logarithmic model, spectral decomposition, linearizations, tangent moduli, finite element method, eversion of tubes.

AMS 2010 subject classification: 74B20, 65N30, 65-04

\footnotetext{
${ }^{1}$ Corresponding author: Boumediene Nedjar, Université Paris-Est, MAST, EMMS, IFSTTAR, Boulevard Newton, 77447 Marne-la-Vallée Cedex 2, France, email: boumediene.nedjar@ifsttar.fr

${ }^{2}$ Herbert Baaser, Mechanical Engineering, University of Applied Sciences Bingen, 55411 Bingen, Germany, email: h.baaser@th-bingen.de

${ }^{3}$ Robert J. Martin, Lehrstuhl für Nichtlineare Analysis und Modellierung, Fakultät für Mathematik, Universität DuisburgEssen, Thea-Leymann Str. 9, 45127 Essen, Germany, email: robert.martin@uni-due.de

${ }^{4}$ Patrizio Neff, Head of Lehrstuhl für Nichtlineare Analysis und Modellierung, Fakultät für Mathematik, Universität DuisburgEssen, Thea-Leymann Str. 9, 45127 Essen, Germany, email: patrizio.neff@yahoo.de
} 


\section{Contents}

1 Introduction 2

1.1 The exponentiated Hencky energy . . . . . . . . . . . . . . . . . . . . 2

1.2 Notation ................................. 5

2 Variational formulation and linearized forms 5

2.1 Linearization of the form $(5) \ldots \ldots \ldots$

2.2 Linearization of the equivalent form $(6) \ldots \ldots \ldots$

2.3 Outlines of the finite element discretization . . . . . . . . . . . . . . . . . 7

2.4 Tangent moduli . . . . . . . . . . . . . . . . . . . . . . . 8

3 Strain-energy based on principal logarithmic stretches 8

3.1 Material tangent modulus . . . . . . . . . . . . . . . . . . . . 10

3.2 Spatial tangent modulus . . . . . . . . . . . . . . . . . . . . . 11

3.3 Mixed tangent modulus . . . . . . . . . . . . . . . . . . . . . . . 11

3.4 Numerical treatment of the case of equal principal stretches . . . . . . . . . . . . . 12

4 Application to the exponentiated Hencky strain energy 13

5 The planar version of the exponentiated Hencky model 15

6 Finite element simulations $\mathbf{1 6}$

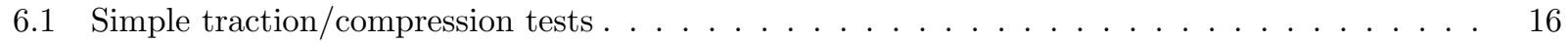

6.2 Footing example with complex loading . . . . . . . . . . . . . . . . . 17

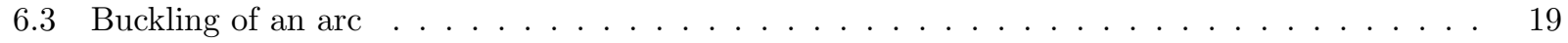

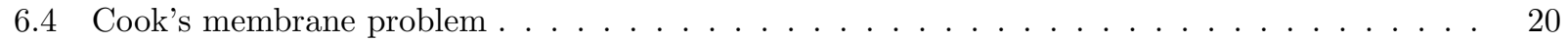

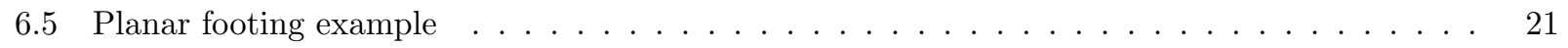

7 Eversion of a tube 24

7.1 Implementation . . . . . . . . . . . . . . . . . . . . . . . . . . 25

7.2 Parameter fitting . . . . . . . . . . . . . . . . . . . . . . . 25

7.3 Simulation and results . . . . . . . . . . . . . . . . . . . . . 26

8 Conclusion and perspectives $\quad 26$

\section{Introduction}

\subsection{The exponentiated Hencky energy}

In a series of articles [34, 35, 32, 11, Neff et al. recently introduced the so-called exponentiated Henckylogarithmic model, a hyperelastic constitutive law induced by the exponentiated Hencky strain energy

$$
\begin{aligned}
\widehat{W}_{\mathrm{eH}}(\boldsymbol{F}) & =\frac{\mu}{k} \exp \left[k\left\|\operatorname{dev}_{n} \log \boldsymbol{U}\right\|^{2}\right]+\frac{\kappa}{2 \hat{k}} \exp \left[\hat{k}(\log \operatorname{det} \boldsymbol{U})^{2}\right] \\
& =\frac{\mu}{k} \exp \left[k\left\|\operatorname{dev}_{n} \log \boldsymbol{V}\right\|^{2}\right]+\frac{\kappa}{2 \hat{k}} \exp \left[\hat{k}(\log \operatorname{det} \boldsymbol{V})^{2}\right] .
\end{aligned}
$$

Here, $\mu>0$ is the (infinitesimal) shear modulus, $\kappa>0$ is the (infinitesimal) bulk modulus, $k$ and $\hat{k}$ are additional dimensionless material parameters determining the strain hardening response, $\boldsymbol{U}=\sqrt{\boldsymbol{F}^{T} \boldsymbol{F}}$ and $\boldsymbol{V}=\sqrt{\boldsymbol{F F}^{T}}$ are the right and left stretch tensor corresponding to the deformation gradient $\boldsymbol{F}$, log denotes 


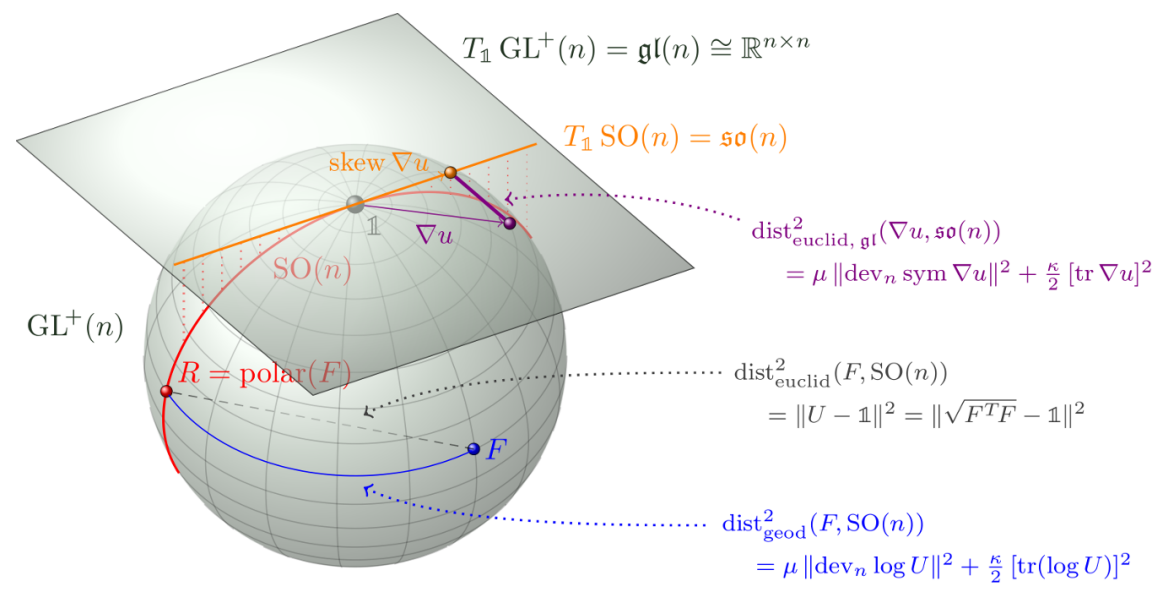

Figure 1: An intuitive comparison between the geodesic and Euclidean distance of $F$ to $\mathrm{SO}(n)$ and the linearized distance of $\nabla u=\boldsymbol{F}-\mathbf{1}$ to the space $\mathfrak{s o}(n)$ of linearized (infinitesimal) rotations.

the principal matrix logarithm on the set of positive definite symmetric matrices, $\operatorname{dev}_{n} \boldsymbol{X}=\boldsymbol{X}-\frac{1}{n}(\operatorname{tr} \boldsymbol{X}) \mathbf{1}$ and $\|\boldsymbol{X}\|=\sqrt{\operatorname{tr} \boldsymbol{X}^{T} \boldsymbol{X}}$ are the deviatoric part and the Frobenius matrix norm of an $n \times n$-matrix $\boldsymbol{X}$, respectively, and tr denotes the trace operator.

The exponentiated Hencky energy is based on the so-called volumetric and isochoric logarithmic strain measures

$$
\omega_{\text {iso }}=\left\|\operatorname{dev}_{n} \log \boldsymbol{U}\right\|=\left\|\operatorname{dev}_{n} \log \boldsymbol{V}\right\| \quad \text { and } \quad \omega_{\mathrm{vol}}=|\operatorname{tr} \log \boldsymbol{U}|=|\log \operatorname{det} \boldsymbol{U}|=|\log \operatorname{det} \boldsymbol{V}|,
$$

which have recently been characterized by a unique geometric property [31, Theorem 3.7]: on the general linear group GL $(n)$ of invertible matrices, the geodesic distance of the isochoric part $\frac{\boldsymbol{F}}{\operatorname{det} \boldsymbol{F}^{1 / n}}$ and the volumetric part $(\operatorname{det} \boldsymbol{F})^{1 / n} \mathbf{1}$ of the deformation gradient to the special orthogonal group $\mathrm{SO}(n)$ are given by

$$
\begin{aligned}
& \operatorname{dist}_{\text {geod }}\left(\frac{\boldsymbol{F}}{(\operatorname{det} \boldsymbol{F})^{1 / n}}, \mathrm{SO}(n)\right)=\left\|\operatorname{dev}_{n} \log \boldsymbol{V}\right\|=\omega_{\text {iso }}, \\
& \operatorname{dist}_{\text {geod }}\left((\operatorname{det} \boldsymbol{F})^{1 / n} \mathbf{1}, \mathrm{SO}(n)\right)=|\log \operatorname{det} \boldsymbol{V}|=\omega_{\mathrm{vol}},
\end{aligned}
$$

if $\mathrm{GL}(n)$ is considered as a Riemannian manifold endowed with the canonical left-invariant Riemannian metric $g$, which is given by 21

$$
g_{\boldsymbol{A}}(\boldsymbol{X}, \boldsymbol{Y})=\left\langle\boldsymbol{A}^{-1} \boldsymbol{X}, \boldsymbol{A}^{-1} \boldsymbol{Y}\right\rangle
$$

for $\boldsymbol{A} \in \mathrm{GL}(n)$ and $\boldsymbol{X}, \boldsymbol{Y} \in \mathfrak{g l}(n)=T_{\boldsymbol{A}} \mathrm{GL}(n)$, where $\langle\boldsymbol{X}, \boldsymbol{Y}\rangle=\boldsymbol{Y}^{T} \boldsymbol{X}$ is the the canonical inner product on the space $\mathfrak{g l}(n)$ of all real $n \times n$-matrices.

This purely geometric observation (which is summarized in Fig. 1) identifies $\omega_{\text {iso }}=\left\|\operatorname{dev}_{n} \log \boldsymbol{V}\right\|$ and $\omega_{\mathrm{vol}}=|\log \operatorname{det} \boldsymbol{V}|$ as the "natural" measures of strain in an elastic deformation, which suggests that an idealized elastic strain energy function may be expressed in terms of these quantities alone 1

A classic example of an energy function depending only on $\omega_{\mathrm{iso}}$ and $\omega_{\mathrm{vol}}$ is the quadratic Hencky energy

$$
W_{\mathrm{H}}(\boldsymbol{F})=\mu\left\|\operatorname{dev}_{n} \log \boldsymbol{U}\right\|^{2}+\frac{\kappa}{2}(\log \operatorname{det} \boldsymbol{U})^{2}=\mu\left\|\operatorname{dev}_{n} \log \boldsymbol{V}\right\|^{2}+\frac{\kappa}{2}(\log \operatorname{det} \boldsymbol{V})^{2}
$$

\footnotetext{
${ }^{1}$ Note that, although every objective and isotropic energy function can be expressed in terms of the (material) logarithmic strain tensor $\log \boldsymbol{U}$ (or the spatial strain tensor $\log \boldsymbol{V}$ ), not every such energy can be expressed in terms of the logarithmic strain measures alone [31.
} 
introduced by Heinrich Hencky in 1929 [12, 13, 14, 30]. While the elasticity model induced by the Hencky energy is, for many materials, in very good agreement with experimental observations for up to moderate strains [34, 1, it also suffers from a number of major shortcomings. For example, the Hencky energy is not able to accurately model the qualitative behaviour of materials under very large deformations, and since it is neither polyconvex nor quasiconvex or rank-one convex [29, there are no known methods available to ensure the existence of energy minimizers for general boundary value problems.

The exponentiated Hencky energy $\widehat{W}_{\mathrm{eH}}$ closely approximates the classical quadratic Hencky energy for small deformations, but provides a more accurate model for larger deformations as well as an improvement with respect to some basic constitutive properties. For example, the induced mapping $\boldsymbol{B} \mapsto \boldsymbol{\sigma}(\boldsymbol{B})$ of the Finger tensor $\boldsymbol{B}=\boldsymbol{F} \boldsymbol{F}^{T}$ to the Cauchy stress tensor $\boldsymbol{\sigma}$ is invertible everywhere [36, 18, 36, 23, 24] (and, in particular, det $\frac{\partial \boldsymbol{\sigma}}{\partial \boldsymbol{B}} \neq 0$ for all positive definite symmetric $\left.\boldsymbol{B}\right) 2^{2}$ In the two-dimensional case, the energy $\widehat{W}_{\mathrm{eH}}$ is also polyconvex [35]. The three-dimensional exponentiated Hencky energy, on the other hand, is not overall rank-one convex, although it is Legendre-Hadamard elliptic in a large neighbourhood of the identity tensor $\mathbf{1 .}$ Moreover, in couplings with multiplicative elasto-plasticity, the computation of the elastic trial step always leads to a rank-one convex problem provided the computation is carried out in a (large) neighbourhood of the yield surface [10, 33. This is true since the elastic domain in that model is always included in its rank-one convexity domain, at any given plastic deformation. This property is not known to hold for other non-elliptic formulations since, in general, the elastic domain is not connected to the rank-one convexity domain.

Similar to the classical Hencky energy, the exponentiated Hencky energy is also determined by only few material parameters which have distinct physical characterizations: while the bulk modulus and the shear modulus, respectively, determine the volumetric and isochoric stress response in the infinitesimal range, the additional dimensionless parameters $k$ and $\hat{k}$ determine the strain hardening response for large deformations. This allows for a very simple fitting of parameter values to new materials without requiring extensive experimental measurements.

Furthermore, materials with zero lateral contraction can be modelled by the exponentiated Hencky energy as well: if the parameters $k, \hat{k}$ are chosen such that $k=\frac{2}{3} \hat{k}$, then the exponentiated Hencky energy can be written as

$$
\widehat{W}_{\mathrm{eH}}(\boldsymbol{F})=\frac{1}{2 k}\left(\frac{E}{1+\nu} \exp \left[k\left\|\operatorname{dev}_{n} \log \boldsymbol{U}\right\|^{2}\right]+\frac{E}{2(1-2 \nu)} \exp \left[\frac{2}{3} k(\log \operatorname{det} \boldsymbol{U})^{2}\right]\right),
$$

where $\nu=\frac{3 \kappa-2 \mu}{2(3 \kappa+\mu)}$ denotes Poisson's ratio and $E=\frac{9 \kappa \mu}{3 \kappa+\mu}$ is Young's modulus. In the special case $\nu=0$, no lateral contraction occurs even for finite strain deformations [34]. This formulation does also not suffer from the deficiencies reported in [6] for volumetric-isochoric splits under simple tension; indeed, for positive nonlinear Poisson number $\nu=-\frac{(\log V)_{22}}{(\log V)_{11}}$, longitudinal extension always implies lateral shortening ${ }^{3}$ A number of further salient features of this formulation have been outlined in 34.

A variant of the exponentiated Hencky energy has previously been applied to so-called tire derived materials, where it was found to be in good agreement with experimental data 25]. For the highly nonlinear equation of state (EOS), which relates pressure to purely volumetric deformations, the exponentiated Hencky model performed particularly well. In [42], the exponentiated Hencky energy has also been formally generalized to the anisotropic setting.

In the following, we consider the finite element implementation of the exponentiated Hencky-logarithmic model, using the spectral decomposition of the different stress and strain tensors 4 Since we also consider

\footnotetext{
${ }^{2}$ The invertibility of the mapping $\boldsymbol{B} \mapsto \boldsymbol{\sigma}(\boldsymbol{B})$ also holds for the volumetric-isochorically decoupled representation of the Neo-Hooke and Mooney-Rivlin energies, which are in use for slightly compressible materials like rubber.

${ }^{3}$ The first use of the definition $\nu=-\frac{(\log V)_{22}}{(\log V)_{11}}$ is due to the famous German scientist W. C. Röntgen [41].

${ }^{4}$ Truesdell remarks on the logarithmic strain that "[b]ecause of the difficulty of calculating the off-diagonal components of $[\log B]$ in terms of the displacement gradient, Hencky's theory is hard to use except in trivial cases." [45 p. 202, (49.4)]
} 
the two-dimensional case, the polyconvexity of the exponentiated Hencky energy allows for a complete wellposedness result in that case: energy minimizers exist, and the solution is contained in the Sobolev space $W^{1, r}\left(\mathcal{B}_{0}\right)$ for any $1 \leq r<\infty$.

We study a number of relevant examples to show the performance of our approach, including the challenging application to the eversion of elastic tubes. This problem was referred to by Truesdel[ $\left[\begin{array}{l}5 \\ {[4]}\end{array}\right.$ to show peculiar properties of nonlinear elasticity and has been experimentally dealt with by Gent and Rivlin 9 . The eversion-of-tubes problem is calculated with the commercial finite element solftware ABAQUs, whereas the other computations were done with our proprietary code and checked against results by ABAQUS.

\subsection{Notation}

Throughout the paper, bold face characters refer to vectors, second- and fourth-order tensorial quantities. In particular, 1 denotes the second-order identity tensor with components $\delta_{i j}\left(\delta_{i j}\right.$ being the Kronecker delta), and $\boldsymbol{I}$ is the fourth-order unit tensor of components $I_{i j k l}=\frac{1}{2}\left(\delta_{i k} \delta_{j l}+\delta_{i l} \delta_{j k}\right)$. The notation $(.)^{T}$ is used for the transpose operator and the scalar product ' $\langle.,$.$\rangle ' is used for double tensor contraction ':', i.e. for$ any second-order tensors $\boldsymbol{A}$ and $\boldsymbol{B},\langle\boldsymbol{A}, \boldsymbol{B}\rangle=\operatorname{tr}\left[\boldsymbol{A} \boldsymbol{B}^{T}\right]=A_{i j} B_{i j}$ where, unless specified, summation on repeated indices is always assumed. The notation $\otimes$ stands for the tensorial product. In components, one has $(\boldsymbol{A} \otimes \boldsymbol{B})_{i j k l}=A_{i j} B_{k l}$, and for any two vectors $\boldsymbol{u}$ and $\boldsymbol{v},(\boldsymbol{u} \otimes \boldsymbol{v})_{i j}=u_{i} v_{j}$. Finally, the dot notation will always designate material time derivative, i.e. $(:) \equiv d(\cdot) / d t$.

\section{Variational formulation and linearized forms}

We consider a solid that occupies the reference configuration $\mathcal{B}_{0}$ with boundary $\partial \mathcal{B}_{0}$. A material particle is identified by its position $\boldsymbol{X} \in \mathcal{B}_{0}$, and we trace its motion by its current position in the spatial configuration $\mathcal{B}_{t}$ as $\boldsymbol{x}=\boldsymbol{\varphi}(\boldsymbol{X}, t) \in \mathcal{B}_{t}$, where $\boldsymbol{\varphi}(., t)$ denotes the deformation map in a time interval $[0, T]$. The deformation gradient is defined as $\boldsymbol{F}=\nabla_{\boldsymbol{X}} \boldsymbol{\varphi}$ where $\nabla_{\boldsymbol{X}}($.$) is the material gradient operator with respect to \boldsymbol{X}$. In the same way, $\nabla_{\boldsymbol{x}}($.$) will designate the spatial gradient operator with respect to \boldsymbol{x}$.

The variational formulation of the local form of the mechanical balance equation plays a central role in the numerical solution of boundary-value problems. In its Lagrangian form, it is equivalent to the following weak form:

$$
\int_{\mathcal{B}_{0}}\left\langle\boldsymbol{S}_{1}, \nabla_{\boldsymbol{X}}(\delta \boldsymbol{\varphi})\right\rangle \mathrm{d} V=G_{\text {ext }}(\delta \boldsymbol{\varphi}),
$$

which must hold for any admissible variation $\delta \boldsymbol{\varphi}$ of deformation. Here, $\boldsymbol{S}_{1}=\frac{\partial W}{\partial \boldsymbol{F}}$ is the first Piola-Kirchhoff stress tensor, $G_{\text {ext }}(\delta \varphi)$ is a short hand notation for the virtual work of external loading assumed to be deformation independent for the sake of simplicity. The left integral term is the internal virtual work. This latter may equivalently be expressed as, see for example [15, 44, 50,

$$
\int_{\mathcal{B}_{0}}\left\langle\boldsymbol{S}_{1}, \nabla_{\boldsymbol{X}}(\delta \boldsymbol{\varphi})\right\rangle \mathrm{d} V=\int_{\mathcal{B}_{0}}\left\langle\boldsymbol{\tau}, \nabla_{\boldsymbol{x}}(\delta \boldsymbol{\varphi})\right\rangle \mathrm{d} V
$$

where $\boldsymbol{\tau}$ is the Kirchhoff stress tensor that is connected to $\boldsymbol{S}_{1}$ by the stress relation $\boldsymbol{\tau}=\boldsymbol{S}_{1} \boldsymbol{F}^{T}{ }^{6}$ Indeed, one

\footnotetext{
${ }^{5}$ An everted rubber tube (shown in Fig. 17 ) was described by Truesdell as follows: "We see that the everted piece is a little longer than the other [identical, non-everted tube]. [...] With the naked eye we can see that the wall of the everted piece is a little thinner than it was originally. If we consider the part of the tube that lies a distance from the ends greater than one-fifth of the diameter, we can say that the everted piece, like the undeformed one, is very nearly a right-circular cylinder. We can idealize what we have seen by saying that an infinitely long, elastic, right-circular cylinder can be turned inside out so as to form another right-circular cylinder, having different radii."

${ }^{6}$ Note that for isotropic materials, the Kirchhoff stress can be obtained directly from the elastic energy as

$$
\boldsymbol{\tau}=\frac{\partial W}{\partial \log \boldsymbol{V}}=\frac{\partial W}{\partial V} \cdot V
$$

a formula first derived by Richter [40, see also 48.
} 
has

$$
\left\langle\boldsymbol{S}_{1}, \nabla_{\boldsymbol{X}}(\delta \boldsymbol{\varphi})\right\rangle=\left\langle\boldsymbol{S}_{1} \boldsymbol{F}^{T}, \nabla_{\boldsymbol{X}}(\delta \boldsymbol{\varphi}) \boldsymbol{F}^{-1}\right\rangle \equiv\left\langle\boldsymbol{\tau}, \nabla_{\boldsymbol{x}}(\delta \boldsymbol{\varphi})\right\rangle,
$$

where the connection $\nabla_{\boldsymbol{X}}(\cdot)=\nabla_{\boldsymbol{x}}(\cdot) \boldsymbol{F}$ for first-order tensors has been used as well.

Different numerical strategies can be employed to solve this nonlinear problem. We choose here to use a high fidelity resolution procedure of the Newton-Raphson type. The above problem needs then to be linearized first, and below are the relevant points of this procedure, which we include in detail for the convenience of the reader.

\subsection{Linearization of the form (5)}

As customary, we denote by $\boldsymbol{u}(\boldsymbol{X})$ the displacement of the particle $\boldsymbol{X} \in \mathcal{B}_{0}$ such that $\boldsymbol{\varphi}(\boldsymbol{X})=\boldsymbol{X}+\boldsymbol{u}(\boldsymbol{X})$. In order to quickly obtain the needed elasticity tangent stiffness tensors, taking the rate form of the expression $(7)_{1}$ we have:

$$
\left\langle\dot{\boldsymbol{S}}_{1}, \nabla_{\boldsymbol{X}}(\delta \boldsymbol{\varphi})\right\rangle=\left\langle\frac{\partial \boldsymbol{S}_{1}}{\partial \boldsymbol{F}}: \dot{\boldsymbol{F}}, \nabla_{\boldsymbol{X}}(\delta \boldsymbol{\varphi})\right\rangle=\left\langle\nabla_{\boldsymbol{X}}(\delta \boldsymbol{\varphi}), \frac{\partial \boldsymbol{S}_{1}}{\partial \boldsymbol{F}}: \nabla_{\boldsymbol{X}} \boldsymbol{v}\right\rangle
$$

where $\boldsymbol{v}=\dot{\boldsymbol{u}}$ is the velocity field.

Replacing the velocity $\boldsymbol{v}$ by the linear increment of displacement $\Delta \boldsymbol{u}$, the linearization of the form (5) about a known state $\boldsymbol{u}=\boldsymbol{u}^{(i)}$ at iteration $(i)$ is then given by

$$
\int_{\mathcal{B}_{0}}\left\langle\nabla_{\boldsymbol{X}}(\delta \boldsymbol{\varphi}), \overline{\mathbf{C}}: \nabla_{\boldsymbol{X}}(\Delta \boldsymbol{u})\right\rangle \mathrm{d} V=G_{\text {ext }}(\delta \boldsymbol{\varphi})-\int_{\mathcal{B}_{0}}\left\langle\boldsymbol{S}_{1}^{(i)}, \nabla_{\boldsymbol{X}}(\delta \boldsymbol{\varphi})\right\rangle \mathrm{d} V,
$$

where the right hand-side represents the residual of the mechanical balance with the first Piola-Kirchhoff stress tensor evaluated with $\boldsymbol{u}^{(i)}$. In the integral of the left hand-side, $\overline{\mathbf{C}}$ is the mixed fourth-order tangent modulus with definition

$$
\overline{\mathbf{C}}=\frac{\partial \boldsymbol{S}_{1}}{\partial \boldsymbol{F}}=\frac{\partial^{2} W}{\partial \boldsymbol{F}^{2}} .
$$

\subsection{Linearization of the equivalent form (6)}

Let us again start with the rate form (8), but this time by invoking the second Piola-Kirchhoff stress tensor $\boldsymbol{S}_{2}$. Recalling that the first and the second Piola-Kirchhoff stress tensors are related by

$$
\boldsymbol{S}_{1}=\boldsymbol{F} \boldsymbol{S}_{2}
$$

we then have

$$
\begin{aligned}
\left\langle\dot{\boldsymbol{S}}_{1}, \nabla_{\boldsymbol{X}}(\delta \boldsymbol{\varphi})\right\rangle=\left\langle\left[\dot{\boldsymbol{F}} \boldsymbol{S}_{2}+\boldsymbol{F} \dot{\boldsymbol{S}}_{2}\right], \nabla_{\boldsymbol{X}}(\delta \boldsymbol{\varphi})\right\rangle & =\left\langle\left[\nabla_{\boldsymbol{x}} \boldsymbol{v} \boldsymbol{F} \boldsymbol{S}_{2}+\boldsymbol{F} \dot{\boldsymbol{S}}_{2}\right], \nabla_{\boldsymbol{X}}(\delta \boldsymbol{\varphi})\right\rangle \\
& =\left\langle\left[\nabla_{\boldsymbol{x}} \boldsymbol{v} \boldsymbol{F} \boldsymbol{S}_{2} \boldsymbol{F}^{T}+\boldsymbol{F} \dot{\boldsymbol{S}}_{2} \boldsymbol{F}^{T}\right] \boldsymbol{F}^{-T}, \nabla_{\boldsymbol{X}}(\delta \boldsymbol{\varphi})\right\rangle \\
& =\left\langle\left[\nabla_{\boldsymbol{x}} \boldsymbol{v} \boldsymbol{\tau}+£_{v} \boldsymbol{\tau}\right], \nabla_{\boldsymbol{x}}(\delta \boldsymbol{\varphi})\right\rangle,
\end{aligned}
$$

where, in the second equality we have used the kinematic relation $\nabla_{\boldsymbol{x}} \boldsymbol{v}=\dot{\boldsymbol{F}} \boldsymbol{F}^{-1}=\boldsymbol{L}$ for the spatial velocity gradient and, in the last equality, we have used the connection $\nabla_{\boldsymbol{x}}(\delta \boldsymbol{\varphi})=\nabla_{\boldsymbol{X}}(\delta \boldsymbol{\varphi}) \boldsymbol{F}^{-1}$, the stress relation $\boldsymbol{\tau}=\boldsymbol{F} \boldsymbol{S}_{2} \boldsymbol{F}^{T}$, and the Lie derivative

$$
£_{v} \boldsymbol{\tau}=\boldsymbol{F} \dot{\boldsymbol{S}}_{2} \boldsymbol{F}^{T},
$$

which is equivalent to the Truesdell rate of the Kirchhoff stress

$$
\frac{d}{d t}^{\mathrm{TR}} \tau=F\left[\frac{d}{d t}\left(F^{-1} \tau F^{-T}\right)\right] F^{T}=\dot{\tau}-\boldsymbol{L} \tau-\tau \boldsymbol{L}^{T} .
$$


This latter can be expressed in terms of the spatial tangent modulus $\widetilde{\mathbf{C}}$ as

$$
£_{v} \boldsymbol{\tau}=\widetilde{\mathbf{C}}: \boldsymbol{D} \equiv \widetilde{\mathbf{C}}: \nabla_{\boldsymbol{x}} \boldsymbol{v},
$$

where the replacement of the spatial strain rates $\boldsymbol{D}$ by $\nabla_{\boldsymbol{x}} \boldsymbol{v}$ is justified since $\widetilde{\mathbf{C}}$ enjoys the symmetry conditions.

Now replacing again the velocity $\boldsymbol{v}$ by the linear increment $\Delta \boldsymbol{u}$, the linearization of the mechanical balance about a known state $\boldsymbol{u}^{(i)}$ at iteration $(i)$ is equivalently given by

$$
\int_{\mathcal{B}_{0}}\left[\nabla_{\boldsymbol{x}}(\Delta \boldsymbol{u}) \boldsymbol{\tau}^{(i)} \cdot \nabla_{\boldsymbol{x}}(\delta \boldsymbol{\varphi})+\operatorname{sym}\left[\nabla_{\boldsymbol{x}}(\delta \boldsymbol{\varphi})\right]: \widetilde{\mathbf{C}}: \operatorname{sym}\left[\nabla_{\boldsymbol{x}}(\Delta \boldsymbol{u})\right]\right] \mathrm{d} V=G_{\text {ext }}(\delta \boldsymbol{\varphi})-\int_{\mathcal{B}_{0}}\left\langle\boldsymbol{\tau}^{(i)}, \nabla_{\boldsymbol{x}}(\delta \boldsymbol{\varphi})\right\rangle \mathrm{d} V,
$$

where, on the left hand-side, the integral is composed of the geometric (first term) and the material (second term) contributions to the linearization, while the right hand-side is the residual of the mechanical balance. The form (14) is equivalent to the one based on $\boldsymbol{S}_{1}$ in eq. (9). Observe further that the form (9) contains one term only in the left hand-side while (14) contains two terms.

In the following, it is the form (14) that will be discretized in view of a finite element implementation. One of the objectives of this paper is to compute $\widetilde{\mathbf{C}}$ for the exponentiated Hencky model. Nevertheless, the mixed fourth-order tensor $\overline{\mathbf{C}}$ defined in 10 will be deduced as well.

\subsection{Outlines of the finite element discretization}

In a finite element context, the displacement is defined at the nodes, see Fig. 2 for an illustration. The interpolations of the reference geometry and the displacement field are completely standard, see e.g. [16, 50, 51] for the exposition of these ideas. Over a typical element $\mathcal{B}_{e}$ they take the form

$$
\boldsymbol{X}_{e}(\boldsymbol{\zeta})=\sum_{A=1}^{n_{\text {node }}^{e}} N^{A}(\boldsymbol{\zeta}) \boldsymbol{X}_{A}^{e}, \quad \boldsymbol{u}_{e}(\boldsymbol{\zeta})=\sum_{A=1}^{n_{\text {node }}^{e}} N^{A}(\boldsymbol{\zeta}) \boldsymbol{u}_{A}^{e},
$$

where $\boldsymbol{X}_{A}^{e} \in \mathbb{R}^{n}$ and $\boldsymbol{u}_{A}^{e} \in \mathbb{R}^{n}$ denote, the reference position and the displacement vector, respectively, that are associated with the element node $A, n=2$ or 3 is the space dimension, $n_{\text {node }}^{e}$ is the node number within the element, and $N^{A}(\boldsymbol{\zeta})$ are the classical isoparametric shape functions.

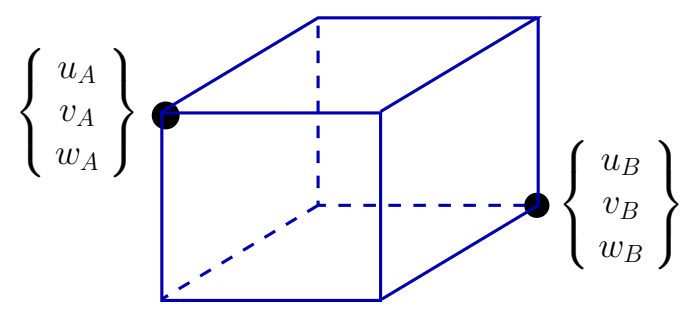

Figure 2: Typical finite element with nodal dofs.

The interpolation over a typical element of the deformation gradient then takes the form

$$
\boldsymbol{F}_{e}(\boldsymbol{\zeta})=\sum_{A=1}^{n_{\text {node }}^{e}}\left(\boldsymbol{X}_{A}^{e}+\boldsymbol{u}_{A}^{e}\right) \otimes \nabla_{\boldsymbol{X}}\left[N^{A}\right], \quad \text { with } \nabla_{\boldsymbol{X}}\left[N^{A}\right]=\mathbf{J}(\boldsymbol{\zeta})^{-T} \nabla_{\boldsymbol{\zeta}}\left[N^{A}\right],
$$


where $\nabla_{\boldsymbol{\zeta}}$ [.] is the gradient relative to the isoparametric coordinates, and where $\mathbf{J}(\boldsymbol{\zeta})=\partial \boldsymbol{X}_{e}(\boldsymbol{\zeta}) / \partial \boldsymbol{\zeta}$ denotes the Jacobian of the isoparametric map $\boldsymbol{\zeta} \rightarrow \boldsymbol{X}$. The finite element discretization of (14) needs the spatial gradients of the shape functions. This is determined via the standard formula

$$
\nabla_{\boldsymbol{x}}\left[N^{A}\right]=\boldsymbol{F}_{e}^{-T} \nabla_{\boldsymbol{X}}\left[N^{A}\right], \quad A=1, \ldots n_{\text {node }}^{e}
$$

The rest of the finite element implementation is completely standard. The element contributions to the global tangent stiffness matrix associated with the element nodes are written as

$$
\mathbf{K}_{e}^{A B}=\int_{\mathcal{B}_{e}} \mathbb{B}^{T}\left[N^{A}\right] \widetilde{\mathbf{C}} \mathbb{B}\left[N^{B}\right] \mathrm{d} V_{e}+\left[\int_{\mathcal{B}_{e}} \nabla_{\boldsymbol{x}}\left[N^{A}\right] \cdot \tau \nabla_{\boldsymbol{x}}\left[N^{B}\right] \mathrm{d} V_{e}\right] \boldsymbol{I}_{n}
$$

for $A, B=1, \ldots n_{\text {node }}^{e}$. In this matrix, $\boldsymbol{I}_{n}$ denotes the $n \times n$ identity matrix, and $\mathbb{B}\left[N^{A}\right]$ is the discrete spatial symmetric gradient operator.

\subsection{Tangent moduli}

One method to reach the theoretical expression of the spatial tangent modulus $\widetilde{\mathbf{C}}$ in the linearized form given by eq. (14) is to proceed in two steps:

- Step (i): we first determine the material tangent modulus $\widehat{\mathbf{C}}$ that is obtained by time derivative of the second Piola-Kirchhoff stress tensor $\boldsymbol{S}_{2}$, and such that

$$
\dot{\boldsymbol{S}}_{2}=\widehat{\mathbf{C}}: \frac{1}{2} \dot{\boldsymbol{C}}
$$

- Step (ii) the spatial tangent modulus $\widetilde{\mathbf{C}}$ is then obtained by push-forward of the above result to the actual configuration as, see eq. $(12), £_{v} \boldsymbol{\tau}=\boldsymbol{F} \dot{\boldsymbol{S}}_{2} \boldsymbol{F}^{T}$ such that

$$
£_{v} \boldsymbol{\tau}=\widetilde{\mathbf{C}}: D
$$

where $\boldsymbol{D}=\operatorname{sym}\left[\dot{\boldsymbol{F}} \boldsymbol{F}^{-1}\right]$ is the spatial strain rate tensor. The useful kinematic relationship

$$
\dot{\boldsymbol{C}}=2 \boldsymbol{F}^{T} \boldsymbol{D} \boldsymbol{F}
$$

is to be employed during the derivation.

This method is valid for any hyperelastic model. We will explicit it here for our case.

\section{Strain-energy based on principal logarithmic stretches}

Let us consider a general elastic model with a strain-energy function $W$ written as a function of the principal logarithmic stretches:

$$
W \equiv W\left(\log \lambda_{1}, \log \lambda_{2}, \log \lambda_{3}\right)
$$

where $\lambda_{1}, \lambda_{2}, \lambda_{3}$ are the principal stretches. The relation with $\widehat{W}_{\mathrm{eH}}$ in $(1)$ will become clear later on.

Furthermore, as spectral decompositions will be employed for the numerical implementation, we recall some basic results and notations. The spectral decompositions of the deformation gradient $\boldsymbol{F}$, the right 
Cauchy-Green tensor $\boldsymbol{C}=\boldsymbol{F}^{T} \boldsymbol{F}$, the left Cauchy-Green tensor $\boldsymbol{B}=\boldsymbol{F} \boldsymbol{F}^{T}$, the right-stretch $\boldsymbol{U}$, and the left-stretch $\boldsymbol{V}$ are respectively given by

$$
\begin{aligned}
& \boldsymbol{F}=\sum_{k=1}^{3} \lambda_{k} \boldsymbol{n}^{(k)} \otimes \boldsymbol{N}^{(k)}, \quad \boldsymbol{C}=\sum_{k=1}^{3} \lambda_{k}^{2} \boldsymbol{N}^{(k)} \otimes \boldsymbol{N}^{(k)}, \quad \boldsymbol{B}=\sum_{k=1}^{3} \lambda_{k}^{2} \boldsymbol{n}^{(k)} \otimes \boldsymbol{n}^{(k)}, \\
& \boldsymbol{U}=\sum_{k=1}^{3} \lambda_{k} \boldsymbol{N}^{(k)} \otimes \boldsymbol{N}^{(k)}, \quad \boldsymbol{V}=\sum_{k=1}^{3} \lambda_{k} \boldsymbol{n}^{(k)} \otimes \boldsymbol{n}^{(k)},
\end{aligned}
$$

where $\boldsymbol{N}^{(k)}$ and $\boldsymbol{n}^{(k)}, k=1,2,3$, are the principal vectors in the material and the spatial configurations, respectively. They are related by

$$
\boldsymbol{F} \boldsymbol{N}^{(k)}=\lambda_{k} \boldsymbol{n}^{(k)}, \quad k=1,2,3 .
$$

Likewise, for the second Piola-Kirchhoff and Kirchhoff stress tensors, we have

$$
\boldsymbol{S}_{2}=\sum_{k=1}^{3} S_{2}^{k} \boldsymbol{N}^{(k)} \otimes \boldsymbol{N}^{(k)}, \quad \boldsymbol{\tau}=\sum_{k=1}^{3} \tau_{k} \boldsymbol{n}^{(k)} \otimes \boldsymbol{n}^{(k)},
$$

where $S_{2}^{k}$ and $\tau_{k}, k=1,2,3$, are the respective principal stresses.

Let us further recall the following useful property of the derivative of the principal stretches with respect to the right Cauchy-Green tensor: In the case of different eigenvalues $\lambda_{1} \neq \lambda_{2} \neq \lambda_{3}$, we have the following result deduced from $22{ }_{2}$ :

$$
\frac{\partial \lambda_{k}^{2}}{\partial \boldsymbol{C}}=\boldsymbol{N}^{(k)} \otimes \boldsymbol{N}^{(k)} .
$$

Proof. Total differentiation of $\boldsymbol{C}$ in eq. $22{ }_{2}$, gives

$$
d \boldsymbol{C}=\sum_{k=1}^{3} 2 \lambda_{k} d \lambda_{k} \boldsymbol{N}^{(k)} \otimes \boldsymbol{N}^{(k)}+\lambda_{k}^{2}\left\{d \boldsymbol{N}^{(k)} \otimes \boldsymbol{N}^{(k)}+\boldsymbol{N}^{(k)} \otimes d \boldsymbol{N}^{(k)}\right\} .
$$

Now recall that $\boldsymbol{N}^{(k)}$ is a unit vector, so that $\boldsymbol{N}^{(k)} \cdot d \boldsymbol{N}^{(k)}=0$. Hence, pre- and post-multiplying $d \boldsymbol{C}$ with $\boldsymbol{N}^{(k)}$ gives

$$
\begin{aligned}
\boldsymbol{N}^{(k)} \cdot d \boldsymbol{C} \boldsymbol{N}^{(k)}= & \sum_{l=1}^{3} 2 \lambda_{l} d \lambda_{l} \underbrace{\boldsymbol{N}^{(k)} \cdot \boldsymbol{N}^{(l)} \otimes \boldsymbol{N}^{(l)} \boldsymbol{N}^{(k)}}_{=1 \text { iff } l=k} \\
& +\sum_{l=1}^{3} \lambda_{l}^{2} \boldsymbol{N}^{(k)} \cdot \underbrace{\left\{d \boldsymbol{N}^{(l)} \otimes \boldsymbol{N}^{(l)}+\boldsymbol{N}^{(l)} \otimes d \boldsymbol{N}^{(l)}\right\} \boldsymbol{N}^{(k)}}_{=0} \\
\equiv & 2 \lambda_{k} d \lambda_{k},
\end{aligned}
$$

which means that

$$
\boldsymbol{N}^{(k)} \otimes \boldsymbol{N}^{(k)}: d \boldsymbol{C}=d\left(\lambda_{k}^{2}\right)
$$

and hence the property (25).

Useful for the following derivations, we find for the second Piola-Kirchhoff stress tensor in 24$]_{1}$

$$
\begin{aligned}
\boldsymbol{S}_{2} \equiv 2 \frac{\partial W}{\partial \boldsymbol{C}} & =\sum_{k=1}^{3} 2 \frac{\partial W}{\partial\left(\log \lambda_{k}\right)} \frac{\partial \log \lambda_{k}}{\partial \boldsymbol{C}}=\sum_{k=1}^{3} 2 \frac{\partial W}{\partial\left(\log \lambda_{k}\right)} \frac{1}{\lambda_{k}} \frac{\partial \lambda_{k}}{\partial \boldsymbol{C}} \\
& =\sum_{k=1}^{3} \frac{1}{\lambda_{k}^{2}} \frac{\partial W}{\partial\left(\log \lambda_{k}\right)} \frac{2 \lambda_{k} \partial \lambda_{k}}{\partial \boldsymbol{C}}=\sum_{k=1}^{3} \underbrace{\frac{1}{\lambda_{k}^{2}} \frac{\partial W}{\partial\left(\log \lambda_{k}\right)}}_{:=S_{2}^{k}} \boldsymbol{N}^{(k)} \otimes \boldsymbol{N}^{(k)},
\end{aligned}
$$


where 25 has been used in the derivative employing the chain rule. We then deduce the spectral decomposition of the Kirchhoff stress tensor $\tau$ in $24{ }_{2}$ as

$$
\boldsymbol{\tau} \equiv \boldsymbol{F} \boldsymbol{S}_{2} \boldsymbol{F}^{T}=\sum_{k=1}^{3} \underbrace{\frac{\partial W}{\partial\left(\log \lambda_{k}\right)}}_{:=\tau_{k}} \boldsymbol{n}^{(k)} \otimes \boldsymbol{n}^{(k)}=\frac{\partial W}{\partial \log \boldsymbol{V}}
$$

where 23 has been used for the push-forward procedure. Observe further the relation between the principal stresses:

$$
\tau_{k}=\lambda_{k}^{2} S_{2}^{k}, \quad k=1,2,3 .
$$

\subsection{Material tangent modulus}

To calculate the material modulus as defined in Step (i), eq. (19), we need the time derivatives of the spectral decompositions 222$)_{2}$ and 24$]_{1}$, both defined in the referential configuration. To do so, we exploit the following observation made in [37]: the time derivative of the eigenvectors of $\boldsymbol{C}$, and hence of $\boldsymbol{S}_{2}$, can be expressed as

$$
\dot{\boldsymbol{N}}^{(k)}=\hat{\bar{\Omega}} \boldsymbol{N}^{(k)}=\sum_{k=1, l \neq k}^{3} \hat{\bar{\Omega}}_{k l} \boldsymbol{N}^{(l)},
$$

where the antisymmetric tensor $\hat{\bar{\Omega}}$ is the spin of the Lagrangian principal axes, i.e. with components $\hat{\bar{\Omega}}_{k l}=$ $-\hat{\bar{\Omega}}_{l k}$. Inserting this result into the time derivative of the spectral decomposition of $C$, eq. $\left.{ }_{22}\right]_{2}$, gives

$$
\begin{aligned}
\frac{1}{2} \dot{\boldsymbol{C}} & =\sum_{k=1}^{3} \frac{1}{2} \frac{d}{d t}\left[\lambda_{k}^{2} \boldsymbol{N}^{(k)} \otimes \boldsymbol{N}^{(k)}\right] \\
& =\sum_{k=1}^{3}\left\{\frac{d}{d t}\left[\frac{1}{2} \lambda_{k}^{2}\right] \boldsymbol{N}^{(k)} \otimes \boldsymbol{N}^{(k)}+\frac{1}{2} \lambda_{k}^{2}\left[\dot{\boldsymbol{N}}^{(k)} \otimes \boldsymbol{N}^{(k)}+\boldsymbol{N}^{(k)} \otimes \dot{\boldsymbol{N}}^{(k)}\right]\right\} \\
& =\sum_{k=1}^{3} \frac{d}{d t}\left[\frac{1}{2} \lambda_{k}^{2}\right] \boldsymbol{N}^{(k)} \otimes \boldsymbol{N}^{(k)}+\sum_{k=1}^{3} \sum_{l=1, l \neq k}^{3} \frac{1}{2}\left(\lambda_{k}^{2}-\lambda_{l}^{2}\right) \hat{\bar{\Omega}}_{k l} \boldsymbol{N}^{(k)} \otimes \boldsymbol{N}^{(l)}
\end{aligned}
$$

Similarly for the time derivative of the spectral decomposition of $\boldsymbol{S}_{2}$ in $24{ }_{1}$, we obtain

$$
\begin{aligned}
\dot{\boldsymbol{S}}_{2}= & \sum_{k=1}^{3} \sum_{l=1}^{3} \frac{1}{\lambda_{l}} \frac{\partial S_{2}^{k}}{\partial \lambda_{l}} \frac{d}{d t}\left[\frac{1}{2} \lambda_{l}^{2}\right] \boldsymbol{N}^{(k)} \otimes \boldsymbol{N}^{(k)} \\
& +\sum_{k=1}^{3} \sum_{l=1, l \neq k}^{3} \frac{S_{2}^{k}-S_{2}^{l}}{\frac{1}{2}\left(\lambda_{k}^{2}-\lambda_{l}^{2}\right)} \frac{1}{2}\left(\lambda_{k}^{2}-\lambda_{l}^{2}\right) \hat{\bar{\Omega}}_{k l} \boldsymbol{N}^{(k)} \otimes \boldsymbol{N}^{(l)}
\end{aligned}
$$

Hence, from the relation (19), we identify the material tangent modulus as

$$
\begin{aligned}
4 \cdot \frac{\partial^{2} W}{\partial \boldsymbol{C}^{2}}=\widehat{\mathbf{C}}= & \sum_{k=1}^{3} \sum_{l=1}^{3} \frac{1}{\lambda_{l}} \frac{\partial}{\partial \lambda_{l}}\left[\frac{1}{\lambda_{k}^{2}} \frac{\partial W}{\partial\left(\log \lambda_{k}\right)}\right] \boldsymbol{N}^{(k)} \otimes \boldsymbol{N}^{(k)} \otimes \boldsymbol{N}^{(l)} \otimes \boldsymbol{N}^{(l)} \\
& +\sum_{k=1}^{3} \sum_{l=1, l \neq k}^{3} \frac{S_{2}^{k}-S_{2}^{l}}{\lambda_{k}^{2}-\lambda_{l}^{2}} \boldsymbol{N}^{(k)} \otimes \boldsymbol{N}^{(l)}\left\{\boldsymbol{N}^{(k)} \otimes \boldsymbol{N}^{(l)}+\boldsymbol{N}^{(l)} \otimes \boldsymbol{N}^{(k)}\right\}
\end{aligned}
$$




\subsection{Spatial tangent modulus}

Observe first that by use of the chain rule, the factor of the first summation in eq. (31) can be rewritten as

$$
\frac{1}{\lambda_{l}} \frac{\partial}{\partial \lambda_{l}}\left[\frac{1}{\lambda_{k}^{2}} \frac{\partial W}{\partial\left(\log \lambda_{k}\right)}\right]=\frac{1}{\lambda_{k}^{2} \lambda_{l}^{2}}\left[\frac{\partial^{2} W}{\partial\left(\log \lambda_{k}\right) \partial\left(\log \lambda_{l}\right)}-2 \delta_{k l} \frac{\partial W}{\partial\left(\log \lambda_{l}\right)}\right] .
$$

Now using the push-forward procedure with the help of (23), the spatial tangent modulus as defined in Step (ii), eq. 20, is given by

$$
\begin{aligned}
\widetilde{\mathbf{C}}= & \sum_{k=1}^{3} \sum_{l=1}^{3}\left[\frac{\partial^{2} W}{\partial\left(\log \lambda_{k}\right) \partial\left(\log \lambda_{l}\right)}-2 \delta_{k l} \tau_{l}\right] \boldsymbol{n}^{(k)} \otimes \boldsymbol{n}^{(k)} \otimes \boldsymbol{n}^{(l)} \otimes \boldsymbol{n}^{(l)} \\
& +\sum_{k=1}^{3} \sum_{l=1, l \neq k}^{3} \underbrace{\frac{\tau_{k} \lambda_{l}^{2}-\tau_{l} \lambda_{k}^{2}}{\lambda_{k}^{2}-\lambda_{l}^{2}}}_{:=\chi} \boldsymbol{n}^{(k)} \otimes \boldsymbol{n}^{(l)}\left\{\boldsymbol{n}^{(k)} \otimes \boldsymbol{n}^{(l)}+\boldsymbol{n}^{(l)} \otimes \boldsymbol{n}^{(k)}\right\} .
\end{aligned}
$$

It is this expression (33) that will be implemented numerically to solve boundary-value problems iteratively, see the discrete form (18). It is a function evaluation involving the derivatives of the strain-energy function $W$ with no particular problems when the principal stretches are different, i.e. when $\lambda_{1} \neq \lambda_{2} \neq \lambda_{3}$. However, in the case of equal principal stretches (or very close from the numerical point of view), the factor in the second summation term in (33), denoted for convenience by $\chi$, will cause numerical troubles as it involves a

division by zero. In this case, special care must be taken and the method we use to circumvent this drawback will be detailed later on in Section 3.4 .

\subsection{Mixed tangent modulus}

Notice that the mixed tangent modulus 10 can be deduced by the rate form of the constitutive relation

$$
\dot{\boldsymbol{S}}_{1}=\overline{\mathbf{C}}: \dot{\boldsymbol{F}} .
$$

For the first Piola-Kirchhoff stress tensor, we have

$$
\boldsymbol{S}_{1}=\sum_{k=1}^{3} S_{1}^{k} \boldsymbol{n}^{(k)} \otimes \boldsymbol{N}^{(k)}
$$

where $S_{1}^{k}, k=1,2,3$, are its principal stresses.

Using the following property of the derivative of the three principal stretches with respect to the deformation gradient in the case of different eigenvalues $\lambda_{1} \neq \lambda_{2} \neq \lambda_{3}$, we have from $22{ }_{1}$ :

$$
\frac{\partial \lambda_{k}}{\partial \boldsymbol{F}}=\boldsymbol{n}^{(k)} \otimes \boldsymbol{N}^{(k)},
$$

which, for a strain-energy function written as a function of the principal logarithmic stretches as given by (21), results in the following expression for $\boldsymbol{S}_{1}$ :

$$
\boldsymbol{S}_{1} \equiv \frac{\partial W}{\partial \boldsymbol{F}}=\sum_{k=1}^{3} \frac{\partial W}{\partial\left(\log \lambda_{k}\right)} \frac{\partial \log \lambda_{k}}{\partial \boldsymbol{F}}=\sum_{k=1}^{3} \frac{\partial W}{\partial\left(\log \lambda_{k}\right)} \frac{1}{\lambda_{k}} \frac{\partial \lambda_{k}}{\partial \boldsymbol{F}}=\sum_{k=1}^{3} \underbrace{\frac{1}{\lambda_{k}} \frac{\partial W}{\partial\left(\log \lambda_{k}\right)}}_{:=S_{1}^{k}} \boldsymbol{n}^{(k)} \otimes \boldsymbol{N}^{(k)},
$$


We then have the following relations between the principal first Piola-Kirchhoff stresses $S_{1}^{k}$ defined in (37), the principal second Piola-Kirchhoff stresses $S_{2}^{k}$, and the principal Kirchhoff stresses $\tau_{k}$ :

$$
S_{1}^{k}=\lambda_{k} S_{2}^{k}, \quad \text { and } \quad \tau_{k}=\lambda_{k} S_{1}^{k}, \quad k=1,2,3 .
$$

Now using the developments that led to the moduli $\widehat{\mathbf{C}}$ and $\widetilde{\mathbf{C}}$, we formally obtain for the above mixed tangent modulus

$$
\begin{aligned}
\frac{\partial^{2} W}{\partial \boldsymbol{F}^{2}}=\overline{\mathbf{C}}= & \sum_{k=1}^{3} \sum_{l=1}^{3}\left[\frac{1}{\lambda_{k} \lambda_{l}} \frac{\partial^{2} W}{\partial\left(\log \lambda_{k}\right) \partial\left(\log \lambda_{l}\right)}-\frac{2}{\lambda_{k}} \delta_{k l} S_{1}^{l}\right] \boldsymbol{n}^{(k)} \otimes \boldsymbol{N}^{(k)} \otimes \boldsymbol{n}^{(l)} \otimes \boldsymbol{N}^{(l)} \\
& +\sum_{k=1}^{3} \sum_{l=1, l \neq k}^{3} \frac{S_{1}^{k} \lambda_{l}-S_{1}^{l} \lambda_{k}}{\lambda_{k}^{2}-\lambda_{l}^{2}} \boldsymbol{n}^{(k)} \otimes \boldsymbol{N}^{(l)}\left\{\boldsymbol{n}^{(k)} \otimes \boldsymbol{N}^{(l)}+\boldsymbol{n}^{(l)} \otimes \boldsymbol{N}^{(k)}\right\} .
\end{aligned}
$$

\subsection{Numerical treatment of the case of equal principal stretches}

For the case in which two or even all three eigenvalues $\lambda_{k}$ are equal, the associated two or three principal stresses are also equal, by isotropy. Precisely, focusing on the spatial tangent modulus, eq. (33), the divided difference term denoted by

$$
\chi=\frac{\tau_{k} \lambda_{l}^{2}-\tau_{l} \lambda_{k}^{2}}{\lambda_{k}^{2}-\lambda_{l}^{2}}
$$

gives us 0/0 and must therefore be determined applying l'Hôspital's rule (see e.g. [15, 37] for similar developments):

$$
\lim _{\lambda_{l} \rightarrow \lambda_{k}} \chi\left(\tau_{k}, \tau_{l}, \lambda_{k}, \lambda_{l}\right)=\lim _{\lambda_{l}^{2} \rightarrow \lambda_{k}^{2}} \frac{\tau_{k} \lambda_{l}^{2}-\tau_{l} \lambda_{k}^{2}}{\lambda_{k}^{2}-\lambda_{l}^{2}}:=\frac{\partial}{\partial\left(\lambda_{k}^{2}\right)}\left(\tau_{k} \lambda_{l}^{2}-\tau_{l} \lambda_{k}^{2}\right)
$$

Evaluation of this latter yields

$$
\frac{\partial}{\partial\left(\lambda_{k}^{2}\right)}\left(\tau_{k} \lambda_{l}^{2}-\tau_{l} \lambda_{k}^{2}\right)=\lambda_{l}^{2} \frac{\partial \tau_{k}}{\partial\left(\lambda_{k}^{2}\right)}-\lambda_{k}^{2} \frac{\partial \tau_{l}}{\partial\left(\lambda_{k}^{2}\right)}+\delta_{k l} \tau_{k}-\tau_{l} .
$$

Using the general result of the derivative of a principal Kirchhoff stress $\tau_{i}$ with respect to a principal stretch $\lambda_{j}$

$$
\frac{\partial \tau_{i}}{\partial\left(\lambda_{j}^{2}\right)}=\frac{1}{2 \lambda_{j}^{2}} \frac{\partial^{2} W}{\partial\left(\log \lambda_{i}\right) \partial\left(\log \lambda_{j}\right)}
$$

into 42, and taking into account the fact that $\delta_{k l}=0$ as the sum is over $l \neq k$ in the second summation in (33), we get the result

$$
\left.\chi \approx \frac{1}{2}\left(\frac{\partial^{2} W}{\partial\left(\log \lambda_{k}\right)^{2}}-\frac{\partial^{2} W}{\partial\left(\log \lambda_{k}\right) \partial\left(\log \lambda_{l}\right)}\right)\right|_{\log \lambda_{l}=\log \lambda_{k}}-\left.\frac{\partial W}{\partial\left(\log \lambda_{l}\right)}\right|_{\log \lambda_{l}=\log \lambda_{k}}
$$

In Summary: for equal eigenvalues, the factor $\chi$ in (33) is replaced by the expression (43), and the expression (33) is hence valid for the three cases: $\lambda_{1} \neq \lambda_{2} \neq \lambda_{3} \neq \lambda_{1}, \lambda_{1}=\lambda_{2} \neq \lambda_{3}$ and $\lambda_{1}=\lambda_{2}=\lambda_{3}$. Notice that from the numerical point of view, equal values means close values to within a prescribed tolerance. 


\section{Application to the exponentiated Hencky strain energy}

To use the relations developed so far for the tangent moduli, we first have to express the strain-energy function (1) in terms of the principal logarithmic stretches, i.e. in the form (21). Observe that

$$
\left.\operatorname{dev}_{3} \log \boldsymbol{U}=\sum_{k=1}^{3}\left(\log \lambda_{k}-\frac{1}{3} \log (\operatorname{det} \boldsymbol{U})\right) \boldsymbol{N}^{(k)} \otimes \boldsymbol{N}^{(k)}=\sum_{k=1}^{3} \log (\operatorname{det} \boldsymbol{U})^{-1 / 3} \lambda_{k}\right) \boldsymbol{N}^{(k)} \otimes \boldsymbol{N}^{(k)} .
$$

Introducing for convenience the so-called modified principal stretches

$$
\bar{\lambda}_{k}=(\operatorname{det} \boldsymbol{U})^{-1 / n} \lambda_{k}=\frac{\lambda_{k}}{\sqrt[n]{\lambda_{1} \lambda_{2} \lambda_{3}}}, \quad k=1, \ldots, n
$$

the scalar product in the first term of $\widehat{W}_{\mathrm{eH}}$ in (1) is simply

$$
\left\langle\operatorname{dev}_{n} \log \boldsymbol{U}, \operatorname{dev}_{n} \log \boldsymbol{U}\right\rangle=\sum_{k=1}^{n}\left(\log \bar{\lambda}_{k}\right)^{2} .
$$

In the second term of $\widehat{W}_{\mathrm{eH}}$ in (1), we have

$$
\log \operatorname{det} \boldsymbol{U}=\operatorname{tr}(\log \boldsymbol{U})=\sum_{k=1}^{n} \log \lambda_{k} .
$$

Then, in terms of the principal logarithmic stretches, the exponentiated Hencky strain-energy function (1) can equivalently be written as

$$
W_{\mathrm{eH}}\left(\log \lambda_{1}, \log \lambda_{2}, \log \lambda_{3}\right)=\frac{\mu}{k} e^{\left[k \sum_{i=1}^{n}\left(\log \bar{\lambda}_{i}\right)^{2}\right]}+\frac{\kappa}{2 \hat{k}} e^{\left[\hat{k}\left(\sum_{i=1}^{n} \log \lambda_{i}\right)^{2}\right]} .
$$

During the computation, we need to calculate the principal Kirchhoff stresses so as to reconstitute the Kirchhoff stress tensor, see eq. (27). They are also needed for the computation of the tangent moduli. For the model at hand we have $\boldsymbol{\tau}=\partial_{\log \boldsymbol{V}} W_{\mathrm{eH}}(\log \boldsymbol{V})$, and hence

$$
\tau_{i}=\frac{\partial W_{\mathrm{eH}}}{\partial\left(\log \lambda_{i}\right)}=2 \mu e^{\left[k \sum_{j=1}^{3}\left(\log \bar{\lambda}_{j}\right)^{2}\right]} \log \bar{\lambda}_{i}+\kappa e^{\left[\hat{k}\left(\sum_{j=1}^{3} \log \lambda_{j}\right)^{2}\right]} \sum_{j=1}^{3} \log \lambda_{j} .
$$

The second derivatives used for the tangent modulus $\widetilde{\mathbf{C}}$, eq. $(33)$, are needed as well as for the treatment of the degenerate case of equal eigenvalues, eq. 43). After a straightforward computation and collecting terms, we obtain for the model 48):

$$
\begin{aligned}
\frac{\partial^{2} W_{\mathrm{eH}}}{\partial\left(\log \lambda_{i}\right) \partial\left(\log \lambda_{j}\right)}= & 2 \mu e^{\left[k \sum_{l=1}^{3}\left(\log \bar{\lambda}_{l}\right)^{2}\right]}\left\{2 k \log \bar{\lambda}_{i} \log \bar{\lambda}_{j}+\delta_{i j}-\frac{1}{3}\right\} \\
& +\kappa e^{\left[\hat{k}\left(\sum_{l=1}^{3} \log \lambda_{l}\right)^{2}\right]}\left\{2 \hat{k}\left(\sum_{l=1}^{3} \log \lambda_{l}\right)^{2}+1\right\}
\end{aligned}
$$

Now for the treatment of the case of equal eigenvalues, the factor $\chi$ from $(43)$ we use numerically is then simply given by

$$
\begin{aligned}
\chi & \approx \mu e^{\left[k \sum_{j=1}^{3}\left(\log \bar{\lambda}_{j}\right)^{2}\right]}-\tau_{k} \\
& =\mu e^{\left[k \sum_{j=1}^{3}\left(\log \bar{\lambda}_{j}\right)^{2}\right]}\left(1-2 \log \bar{\lambda}_{k}\right)-\kappa e^{\left[\hat{k}\left(\sum_{j=1}^{3} \log \lambda_{j}\right)^{2}\right]} \sum_{j=1}^{3} \log \lambda_{j},
\end{aligned}
$$


Table 1: Local computation of the contributions to the tangent stiffness and residual.

Given the updated displacements $\boldsymbol{u}^{(i)}$ at iteration $(i)$,

1. Compute the new updated deformation gradient $\boldsymbol{F}$ and, hence, the corresponding left Cauchy-Green tensor $\boldsymbol{B}=\boldsymbol{F} \boldsymbol{F}^{T}=\boldsymbol{V}^{2}$

2. Spectral decomposition of $\boldsymbol{B}$ :

Use the Jacobi method to find the principal stretches $\lambda_{k}$ together with the principal directions $\boldsymbol{n}^{(k)}, k=1,2,3$

3. With the logarithmic stretches, compute the principal Kirchhoff stresses $\tau_{k}$ with the help of 49 and reconstitute the stress tensor as

$$
\boldsymbol{\tau}=\sum_{k=1}^{3} \tau_{k} \boldsymbol{n}^{(k)} \otimes \boldsymbol{n}^{(k)}
$$

4. Compute the second derivatives with the help of (50) together with the divided difference terms $\chi$ whose expression is given by

$$
\chi=\frac{\tau_{k} \lambda_{l}^{2}-\tau_{l} \lambda_{k}^{2}}{\lambda_{k}^{2}-\lambda_{l}^{2}} \quad \text { if } \lambda_{k} \neq \lambda_{l}
$$

or by the limiting value (51) if $\lambda_{k}=\lambda_{l}$,

for $k, l=1,2,3$ and $k \neq l$.

5. Reconstitute the spatial tangent modulus as

$$
\begin{aligned}
\widetilde{\mathbf{C}}= & \sum_{k=1}^{3} \sum_{l=1}^{3}\left[\frac{\partial^{2} W_{\mathrm{eH}}}{\partial\left(\log \lambda_{k}\right) \partial\left(\log \lambda_{l}\right)}-2 \delta_{k l} \tau_{l}\right] \boldsymbol{n}^{(k)} \otimes \boldsymbol{n}^{(k)} \otimes \boldsymbol{n}^{(l)} \otimes \boldsymbol{n}^{(l)} \\
& +\sum_{k=1}^{3} \sum_{l=1, l \neq k}^{3} \chi \boldsymbol{n}^{(k)} \otimes \boldsymbol{n}^{(l)}\left\{\boldsymbol{n}^{(k)} \otimes \boldsymbol{n}^{(l)}+\boldsymbol{n}^{(l)} \otimes \boldsymbol{n}^{(k)}\right\}
\end{aligned}
$$

where the results $(49)$ and $(50)$ have been used.

In the finite element context, the above quantities are computed at the integration points level. For the sake of clarity, the steps involved in this local procedure are summarized in Table 1. Notice that for the spatial configuration we use, we only need the computation of the set of principal vectors $\boldsymbol{n}^{(k)}, k=1,2,3$ together with the corresponding set of principal stretches $\lambda_{k}, k=1,2,3$. We perform for this a spectral decomposition of the left Cauchy-Green tensor, eq. $22{ }_{3}$, by using the classical Jacobi method that gives both sets at the same time.

Remark 1. By taking $k=0$ and $\hat{k}=0$, the principal Kirchhoff stresses (49) become

$$
\tau_{i}=2 \mu \log \bar{\lambda}_{i}+\kappa \sum_{l=1}^{3} \log \lambda_{l},
$$

which is the expression obtained with the classical quadratic Hencky model

$$
W_{\mathrm{H}}=\mu\left\|\operatorname{dev}_{n} \log \boldsymbol{U}\right\|^{2}+\frac{\kappa}{2}[\operatorname{tr}(\log \boldsymbol{U})]^{2}=\mu\left\|\operatorname{dev}_{n} \log \boldsymbol{V}\right\|^{2}+\frac{\kappa}{2}[\operatorname{tr}(\log \boldsymbol{V})]^{2} .
$$

This nowadays classical model has indeed been extensively studied in the literature, see e.g. [1, 43, 38, in finite strain elasticity. Among others, it is also amenable for an easy extension to include other phenomena 
such as finite strain elastoplasticity [2, 43, finite viscoelasticity [26, 39, and growth in biomechanics [27] to mention but a few.

In the case $(k, \hat{k})=(0,0)$, the second derivatives 50 are easily shown to reduce to

$$
\frac{\partial^{2} W_{\mathrm{H}}}{\partial\left(\log \lambda_{i}\right) \partial\left(\log \lambda_{j}\right)}=2 \mu\left\{\delta_{i j}-\frac{1}{3}\right\}+\kappa,
$$

and for equal principal stretches, $\lambda_{k} \approx \lambda_{l}$, we find from (52) and (54) into 443):

$$
\chi \approx \mu\left(1-2 \log \bar{\lambda}_{k}\right)-\kappa \sum_{j=1}^{n} \log \lambda_{j}=\mu-\tau_{k} .
$$

\section{The planar version of the exponentiated Hencky model}

We consider in this section the planar version of the above exponentiated Hencky model, see [11, 35]. In this case $n=2$ and the strain energy function is explicited as

$$
W_{\mathrm{eH}}\left(\log \lambda_{1}, \log \lambda_{2}\right)=\frac{\mu}{k} e^{\left[k\left(\left(\log \bar{\lambda}_{1}\right)^{2}+\left(\log \bar{\lambda}_{2}\right)^{2}\right)\right]}+\frac{\kappa}{2 \hat{k}} e^{\left[\hat{k}\left(\log \lambda_{1}+\log \lambda_{2}\right)^{2}\right]},
$$

where $\bar{\lambda}_{i}=(\operatorname{det} \boldsymbol{U})^{-1 / 2} \lambda_{i}, i=1,2$. Here we suppose the plane $x_{1}-x_{2}$ spanned by the basis $\left\{\vec{e}_{1}, \vec{e}_{2}\right\}$. Note that the planar exponentiated Hencky energy is not the three-dimensional Hencky energy evaluated at planar strain $\sqrt{7}$

The principal Kirchhoff stresses are given by

$$
\tau_{i}=\frac{\partial W_{\mathrm{eH}}}{\partial\left(\log \lambda_{i}\right)}=2 \mu e^{\left[k\left(\left(\log \bar{\lambda}_{1}\right)^{2}+\left(\log \bar{\lambda}_{2}\right)^{2}\right)\right]} \log \bar{\lambda}_{i}+\kappa e^{\left[\hat{k}\left(\log \lambda_{1}+\log \lambda_{2}\right)^{2}\right]}\left(\log \lambda_{1}+\log \lambda_{2}\right) .
$$

and the second derivatives of the strain energy $(56)$ are deduced as

$$
\begin{aligned}
\frac{\partial^{2} W_{\mathrm{eH}}}{\partial\left(\log \lambda_{i}\right) \partial\left(\log \lambda_{j}\right)}= & 2 \mu e^{\left[k\left(\left(\log \bar{\lambda}_{1}\right)^{2}+\left(\log \bar{\lambda}_{2}\right)^{2}\right)\right]}\left\{2 k \log \bar{\lambda}_{i} \log \bar{\lambda}_{j}+\delta_{i j}-\frac{1}{2}\right\} \\
& +\kappa e^{\left[\hat{k}\left(\log \lambda_{1}+\log \lambda_{2}\right)^{2}\right]}\left\{2 \hat{k}\left(\log \lambda_{1}+\log \lambda_{2}\right)^{2}+1\right\}
\end{aligned}
$$

Now for the treatment of the case of equal eigenvalues, $\lambda_{1} \approx \lambda_{2}$, the factor $\chi$ from 443 we use numerically is simply given by

$$
\begin{aligned}
\chi & \approx \mu e^{\left[k\left(\left(\log \bar{\lambda}_{1}\right)^{2}+\left(\log \bar{\lambda}_{2}\right)^{2}\right)\right]}-\tau_{k} \\
& =\mu e^{\left[k\left(\left(\log \bar{\lambda}_{1}\right)^{2}+\left(\log \bar{\lambda}_{2}\right)^{2}\right)\right]}\left(1-2 \log \bar{\lambda}_{k}\right)-\kappa e^{\left[\hat{k}\left(\log \lambda_{1}+\log \lambda_{2}\right)^{2}\right]}\left(\log \lambda_{1}+\log \lambda_{2}\right),
\end{aligned}
$$

where the results (57) and (58) have been used.

\footnotetext{
${ }^{7}$ The restriction of the three-dimensional exponentiated Hencky energy to planar strain is not polyconvex, while $(56)$ is. The difference stems from the definition of the two-dimensional isochoric stretches $\bar{\lambda}_{k}=(\operatorname{det} \boldsymbol{U})^{-1 / 2} \lambda_{k}$.
} 


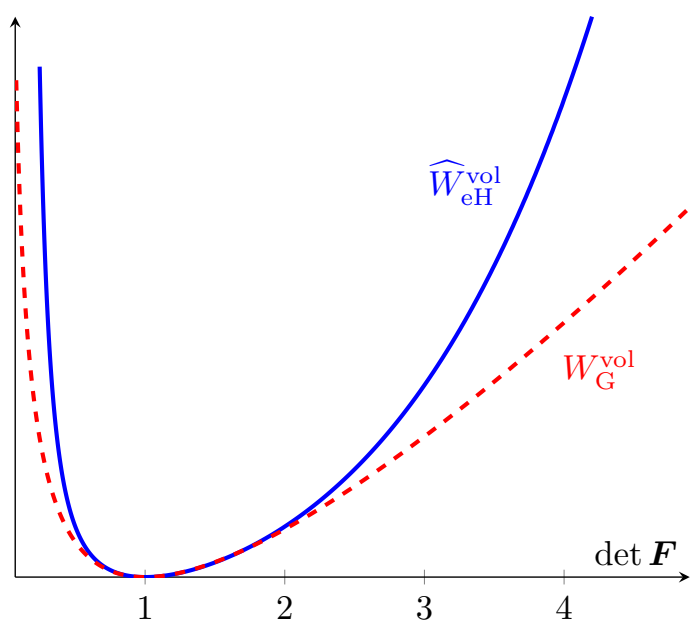

Figure 3: The volumetric part $\widehat{W}_{\mathrm{eH}}^{\mathrm{vol}}=\frac{\kappa}{2 \hat{k}} e^{\hat{k}[\operatorname{det} \boldsymbol{F}]^{2}}$ of the exponentiated Hencky energy compared to the volumetric part $W_{\mathrm{G}}^{\mathrm{vol}}=\frac{3}{8} \kappa\left((\operatorname{det} \boldsymbol{F})^{4 / 3}+\frac{2}{(\operatorname{det} \boldsymbol{F})^{2 / 3}}-3\right)$ of the Gent energy.

\section{$6 \quad$ Finite element simulations}

In this section, some illustrative finite element simulations are performed to highlight the applicability and efficiency of the numerical method developed in the above through two- and three-dimensional problems. For the purpose of comparison, some computations are also made with the classical quadratic Hencky model $W_{\mathrm{H}}$ of eq. (53), a compressible Neo-Hookean model given by, see [28],

$$
W_{\mathrm{nHK}}=\frac{1}{2} \mu\left(\left\|\frac{\boldsymbol{F}}{(\operatorname{det} \boldsymbol{F})^{1 / 3}}\right\|^{2}-3\right)+\frac{3}{8} \kappa\left((\operatorname{det} \boldsymbol{F})^{4 / 3}+\frac{2}{(\operatorname{det} \boldsymbol{F})^{2 / 3}}-3\right),
$$

where we recall that $\operatorname{det} \boldsymbol{U}=\operatorname{det} \boldsymbol{F}$, and a compressible version of the Gent model, see for example [8], whose strain-energy function is here chosen as

$$
W_{\mathrm{G}}=-\frac{J_{m}}{2} \mu \log \left(1-\frac{\left\|\frac{\boldsymbol{F}}{(\operatorname{det} \boldsymbol{F})^{1 / 3}}\right\|^{2}-3}{J_{m}}\right)+\frac{3}{8} \kappa\left((\operatorname{det} \boldsymbol{F})^{4 / 3}+\frac{2}{(\operatorname{det} \boldsymbol{F})^{2 / 3}}-3\right),
$$

where the non-dimensional constant $J_{m}$ denotes the limiting extensibility parameter of the molecular network. This term introduces a singularity when $\left\|\frac{\boldsymbol{F}}{(\operatorname{det} \boldsymbol{F})^{1 / 3}}\right\|^{2}=J_{m}+3$, which provides an accurate representation of the stiffening of rubber near ultimate (elastic) elongation.

Observe that for these two latter models, the strain-energy function is also additively split into a volumepreserving part that depends on the modified deformation gradient $\left(\operatorname{det} \boldsymbol{F}^{-1 / 3}\right) \boldsymbol{F}$, as originally proposed by Richter [40], see also Flory [7, and a volumetric part that depends solely on the Jacobian determinant of the deformation gradient. Moreover, the same volumetric-energy function has been used for both of the expressions $W_{\mathrm{nHK}}$ and $W_{\mathrm{G}}$. For more details about the properties of the model [60), see [28].

\subsection{Simple traction/compression tests}

We consider a $(20 \times 20 \times 20) \mathrm{mm}^{3}$ cubic sample. In this first series of computations, simple traction and compression simulations are performed. As the loading is uniform, it is sufficient to use a coarse mesh, here 
Table 2: Material parameters for the four models.

\begin{tabular}{|l|l|}
\hline Model & Material parameters \\
\hline exponentiated Hencky $W_{\mathrm{eH}}$, eq. (1) or (48) & $\mu, \quad \kappa=4.7 \mu, \quad k=2, \quad \hat{k}=3$ \\
quadratic Hencky $W_{\mathrm{H}}$, eq. (53) & $\mu, \quad \kappa=4.7 \mu$ \\
compressible Neo-Hooke $W_{\mathrm{nHK}}$, eq. 600 & $\mu, \quad \kappa=4.7 \mu$ \\
compressible Gent $W_{\mathrm{G}}$, eq. 61] & $\mu, \quad \kappa=4.7 \mu, \quad J_{m}=5$ \\
\hline
\end{tabular}

for illustration with 64 cubic elements using linear interpolation, i.e. a total of 125 nodes with 375 degrees of freedom.

Now to make matters as concrete as possible, the elastic properties we use for the four models are summarized in Table 2 where the same infinitesimal compressibility parameter $\kappa$ is assumed. Indeed, in the limiting case of linear elasticity, one has for the infinitesimal Poisson's ration $\nu$,

$$
\nu=\frac{3 \kappa-2 \mu}{6 \kappa+2 \mu}
$$

so that with a ratio $\kappa / \mu=4.7$ as in Table 2 , we have $\nu \approx 0.4$ in all of the four cases.

Fig. 4 shows the results with the four models. For the computations, a constant increment of vertical displacement $\Delta \bar{w}_{\mathrm{imp}}=1 \mathrm{~mm}$ has been used. The compression has been computed until $\bar{w}_{\mathrm{imp}}=-15 \mathrm{~mm}$, so until a contraction $\lambda_{3}=0.25$, while the traction has been computed until $\bar{w}_{\text {imp }}=70 \mathrm{~mm}$, so a stretch in extension of $\lambda_{3}=4.5 \equiv 450 \%$. The ordinate axis in Fig. 4 corresponds to the component of nominal stress in the loading direction $\vec{e}_{3}$ which is principal in the present case. The stress is here nondimensionalized with the shear modulus, i.e. $S_{1}^{3} / \mu$.

One can observe the characteristic stiffening exhibited by the exponentiated Hencky model for large stretches in tension as stated, for instance in 34, and similarly so for the Gent model. We also retrieve the well-known non-stiffening characteristics of both the classical quadratic Hencky and Neo-Hookean models in tension. For illustrative purposes, deformed shapes of the sample obtained with the exponentiated Hencky model are also plotted at scale 1 in Fig. 4 .

\subsection{Footing example with complex loading}

This second example is the one of a non uniform loading. It corresponds to the footing example where the above sample is this time subject to a compressive loading on one-half of the top edge while the lateral edges are fixed in their respective normal directions. Here we use a finer mesh with 4096 linear cubic elements, i.e. with 4913 nodes and 14739 degrees of freedom.

For the material parameters, we still use the ones of compressible hyperelasticity given in Table 2 with, this time, $\mu=1 \mathrm{MPa}$ that corresponds to a soft matter with a Young's modulus $E=2.8 \mathrm{MPa}$ when a Poisson's ratio $\nu=0.4$ is used for the limiting case of linear elasticity.

Fig. 5 shows the results of the computations with the four models. The loading increment on the partial top face was always taken constant with value $\Delta \bar{w}_{\text {imp }}=-1 \mathrm{~mm}$. A maximum of 5 iterations were needed for very distorted shapes with the exponentiated Hencky model. As an illustration, the deformed finite element mesh obtained with this latter at $\bar{w}_{\text {imp }}=-8 \mathrm{~mm}$ has been superimposed in Fig. 5 . 


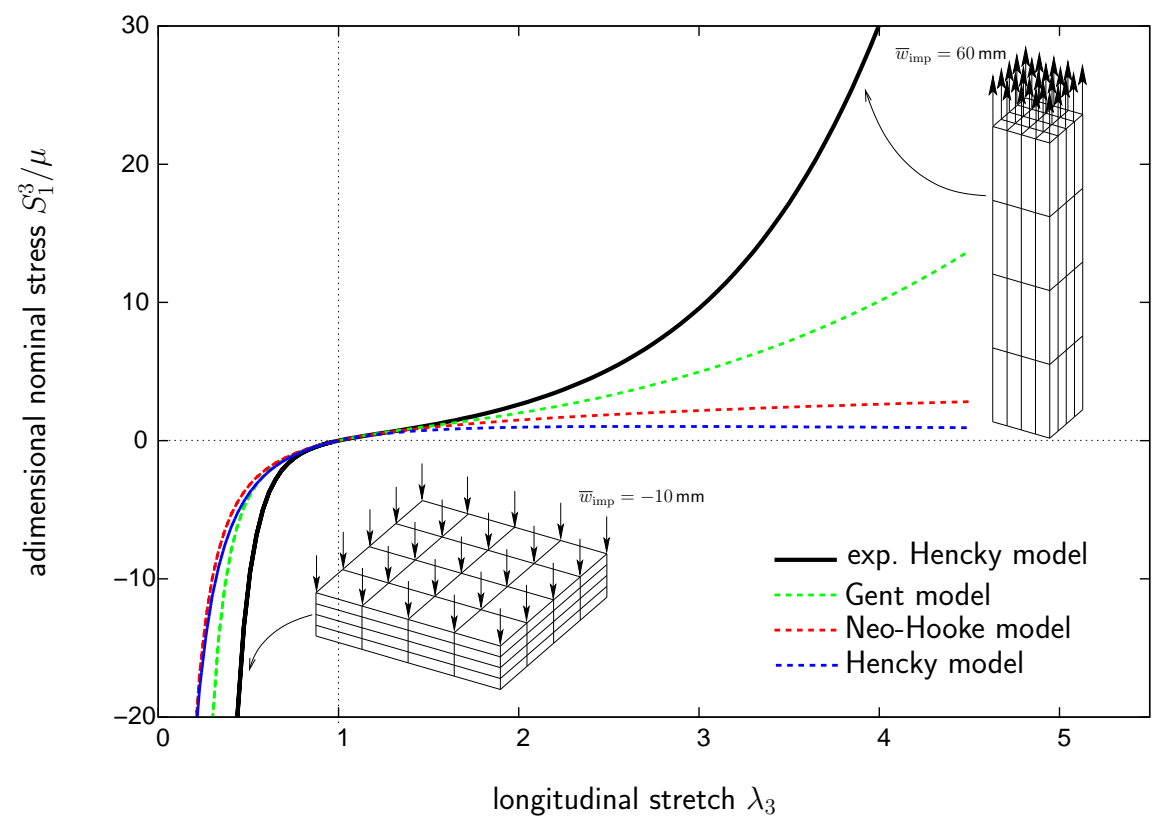

Figure 4: Stress-strain curves with the four models under simple traction/compression. An illustration of deformed configurations obtained with the exponentiated Hencky model.

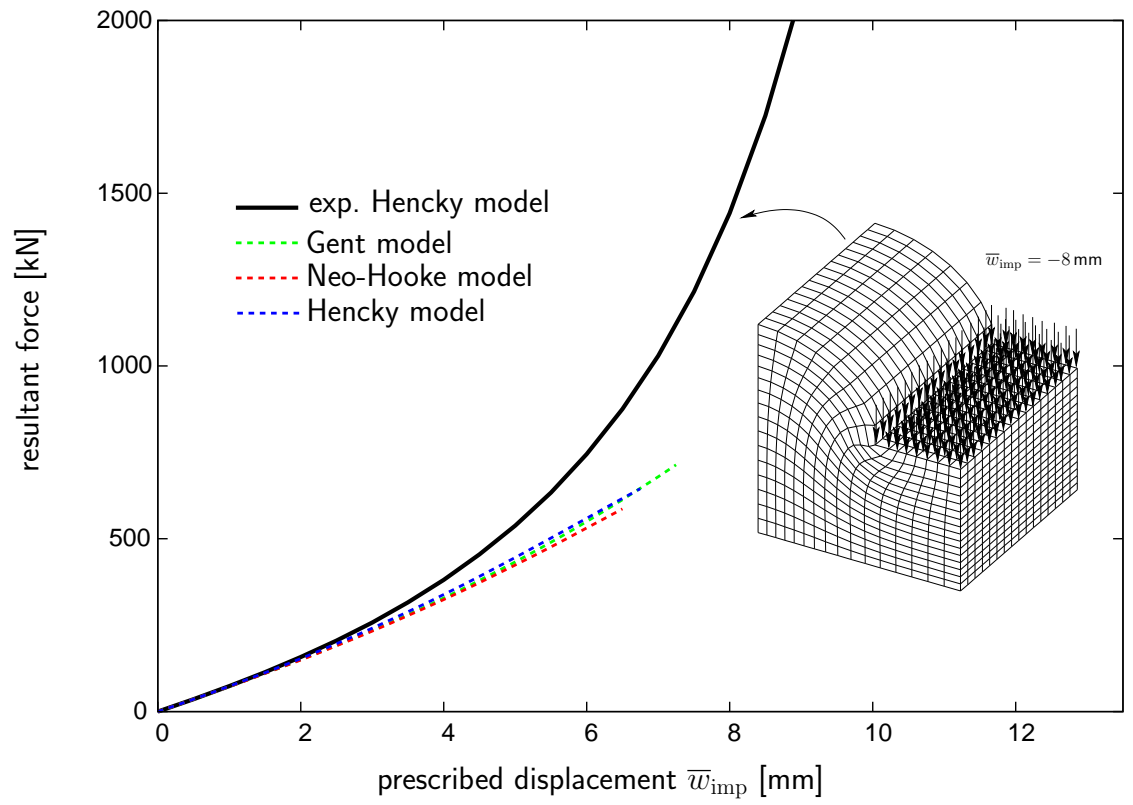

Figure 5: Footing example. Resultant curves for the four models. Deformed configurations obtained with the exponentiated Hencky model at prescribed displacement $\bar{w}_{\mathrm{imp}}=-8 \mathrm{~mm}$. 
In Fig. 6, we show the deformed configurations together with the vertical displacement fields computed with the four models at prescribed displacement $\bar{w}_{\text {imp }}=-6 \mathrm{~mm}$ and, in Fig. 7), we show the same result at $\bar{w}_{\text {imp }}=-12 \mathrm{~mm}$ for only the exponentiated Hencky model. In particular, observe for this loading the very distorted shape of the sample that proves that the numerical implementation of the exponentiated Hencky-logarithmic model is robust.

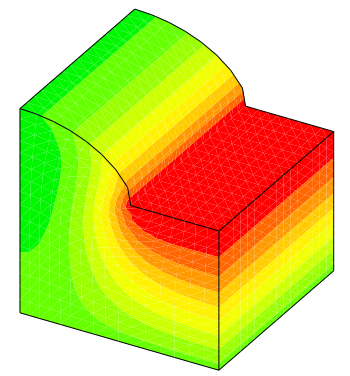

(a)

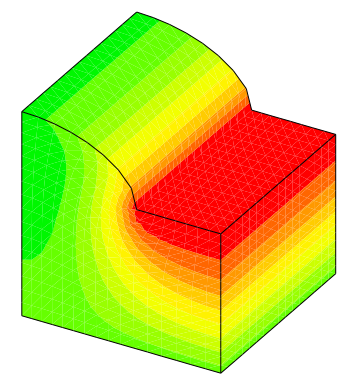

(b)

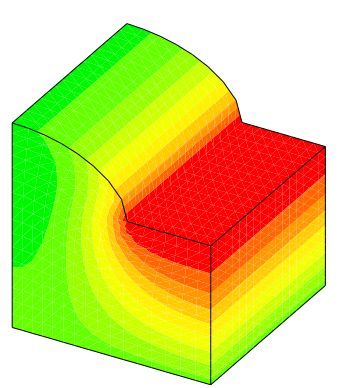

(c)

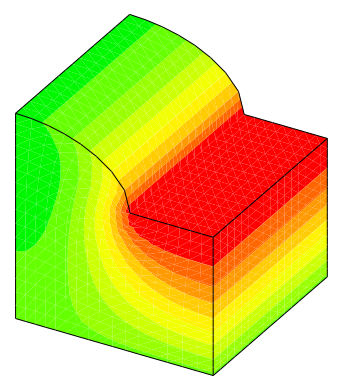

(d)

Figure 6: Deformed configurations and vertical displacement fields at $\bar{w}_{\text {imp }}=-6 \mathrm{~mm}$ with: (a) the exponentiated Hencky model, (b) the Gent model, (c) the Neo-Hookean model, and (d) the quadratic Hencky model.

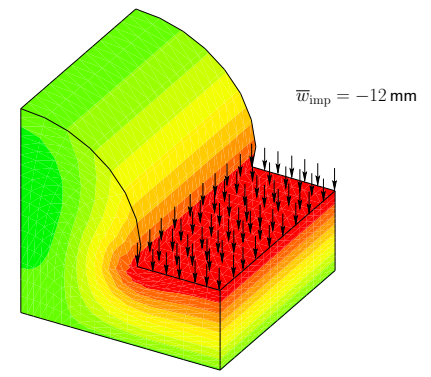

Figure 7: Deformed configuration and vertical displacement field at $\bar{w}_{\text {imp }}=-12 \mathrm{~mm}$ with the exponentiated Hencky model.

\subsection{Buckling of an arc}

In this example, the computation is performed by using the planar 2D-exponentiated Hencky version of the model recalled in Section 5. We consider an arc which spans a width related to an angle of $\alpha=60^{\circ}$. The inner radius of the arc is $R_{\mathrm{i}}=100 \mathrm{~mm}$ and its thickness is $t=4 \mathrm{~mm}$. The arc is clamped at both sides. To show the behavior of the finite element implementation, three mesh refinements with quadrilateral linear elements are used with growing densities, see Fig. 8 .

- mesh 1: three elements used in the thickness direction, and a total of 90 elements corresponding to 248 degrees of freedom.

- mesh 2: 10 elements in the thickness direction, and a total of 900 elements corresponding to 2002 degrees of freedom. 
- mesh 3: 20 elements in the thickness direction, and a total of 3600 elements corresponding to 7602 degrees of freedom.

The material parameters we use are those of Table 2 with $\mu=1 \mathrm{MPa}$.

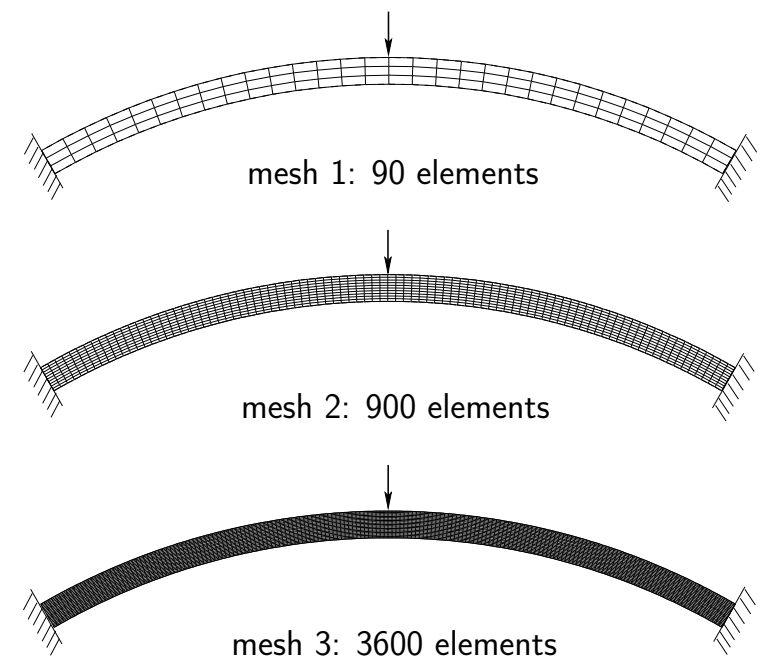

Figure 8: Buckling of a clamped arc. Finite element discretizations used with the planar 2D-exponentiated Hencky model.

The bending load consists on prescribing an increasing vertical displacement $\bar{v}_{\text {imp }}$ of the point-load, i.e. the middle node of the upper edge. For each computation, the same increment $\Delta \bar{v}_{\text {imp }}=0.25 \mathrm{~mm}$ has been used downwards. The three resulting curves are depicted in Fig. 9 as reactive forces versus imposed displacements.

One can observe the good convergence properties. The two denser meshes show close responses while mesh 1 gives a higher peak-load. For illustrative purposes, the deformed mesh 1 at the buckling load and the deformed mesh 3 in a post-buckling configuration are shown in Fig. 9.

\subsection{Cook's membrane problem}

The numerical implementation is tested in this example with the so-called Cook's membrane benchmark problem, which is a classical bending dominated test introduced here to assess element performances with respect to volumetric locking for pertinent simulations, see e.g. [22]. This test consists in a tapered plate clamped on the left side and a uniformly distributed load $F$ is applied on the right free side, see the illustration of the geometry and boundary conditions in Fig. 10. The properties for the planar 2D-exponentiated Hencky model we use are:

$$
\mu=1 \mathrm{MPa}, \quad k=2, \quad \hat{k}=3,
$$

and for the bulk modulus, we perform the test with two different values:

$$
\kappa=4.7 \mathrm{MPa} \text {, and } \kappa=50 \mathrm{MPa} .
$$

The first one corresponds to a compressible hyperelastic model with Poisson's ratio $\nu \approx 0.4$ in the limiting case of linear elasticity, e.g. see eq. (58), while the second one corresponds to quasi-incompressibility with $\nu \approx 0.49$.

In all the computations, the distributed load $F$ has been applied in ten equal increments $\Delta F=20$. Fig. 10 shows the results of the convergence of the vertical displacement of the node $A$ located at the middle of 


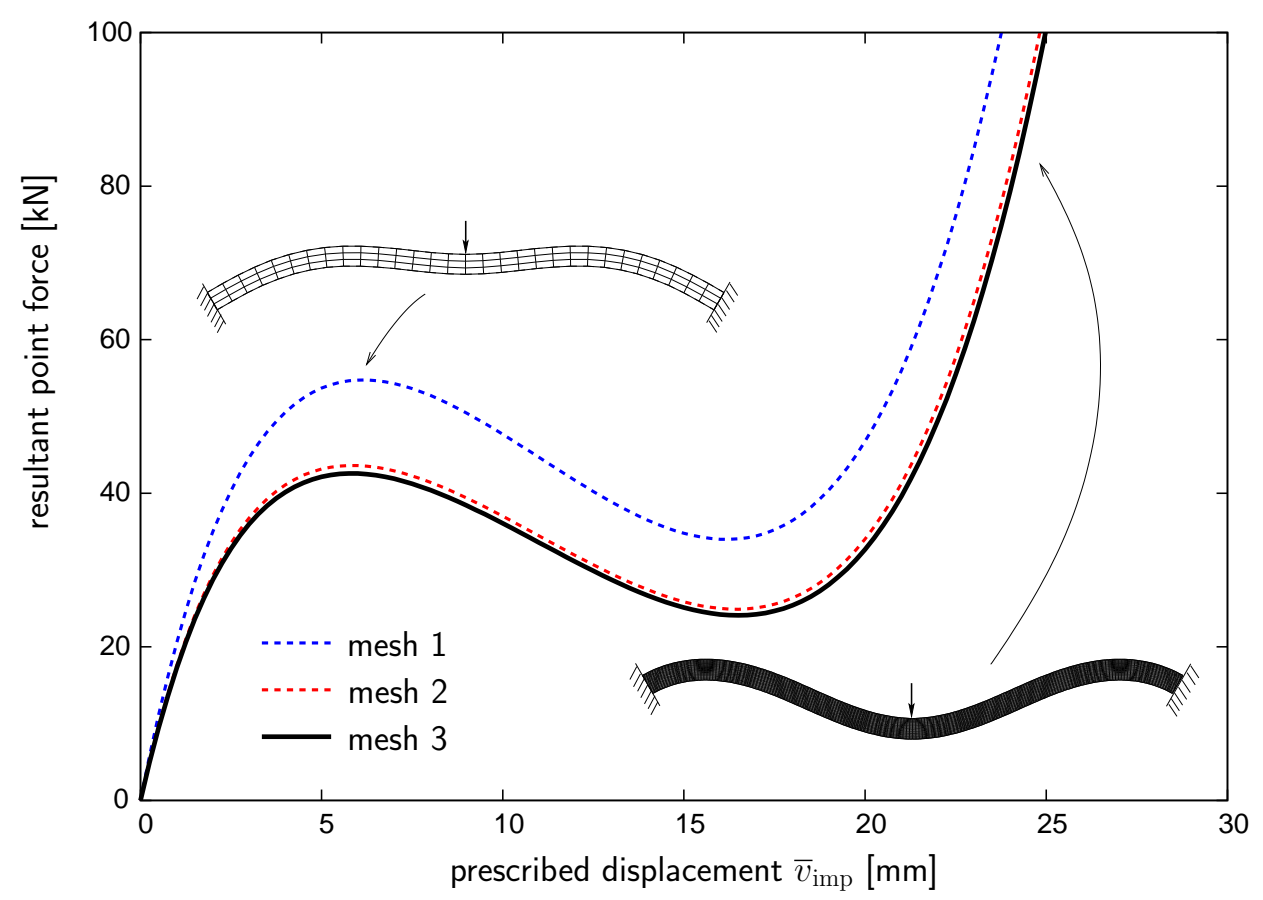

Figure 9: Load/displacement curves of the arc with deformed configurations with the different mesh refinements.

the right edge. One can observe the good convergence properties of the present implementation, even with linear isoparametric elements. For illustrative purposes, Fig. 11 shows the deformed configurations together with the vertical displacement fields for both the compressible and quasi-incompressible material behaviour.

\subsection{Planar footing example}

In this example, we come back to the footing example of Section 6.2 this time within a purely planar analysis. We consider a $20 \times 20 \mathrm{~mm}^{2}$ square sample by using two mesh refinements, see Fig. 12 for the geometry and boundary conditions:

- mesh 1: a coarse mesh with $10 \times 10$ quadrilateral linear elements.

- mesh 2: a finer mesh with $30 \times 30$ quadrilateral linear elements.

The material parameters we use are those of the compressible Cook's membrane that we recall here:

$$
\mu=1 \mathrm{MPa}, \quad \kappa=4.7 \mathrm{MPa}, \quad k=2, \quad \hat{k}=3 .
$$

Fig. 13 shows the results of the two computations. For both meshes, the loading increment on the partial top face was always taken constant with a prescribed value $\Delta \bar{v}_{\text {imp }}=-0.5 \mathrm{~mm}$. A maximum of 5 iterations were needed for very distorted shapes. As an illustration, the deformed finite element meshes obtained with the two computations at prescribed displacement $\bar{v}_{\text {imp }}=-12 \mathrm{~mm}$ are shown in Fig. 14 


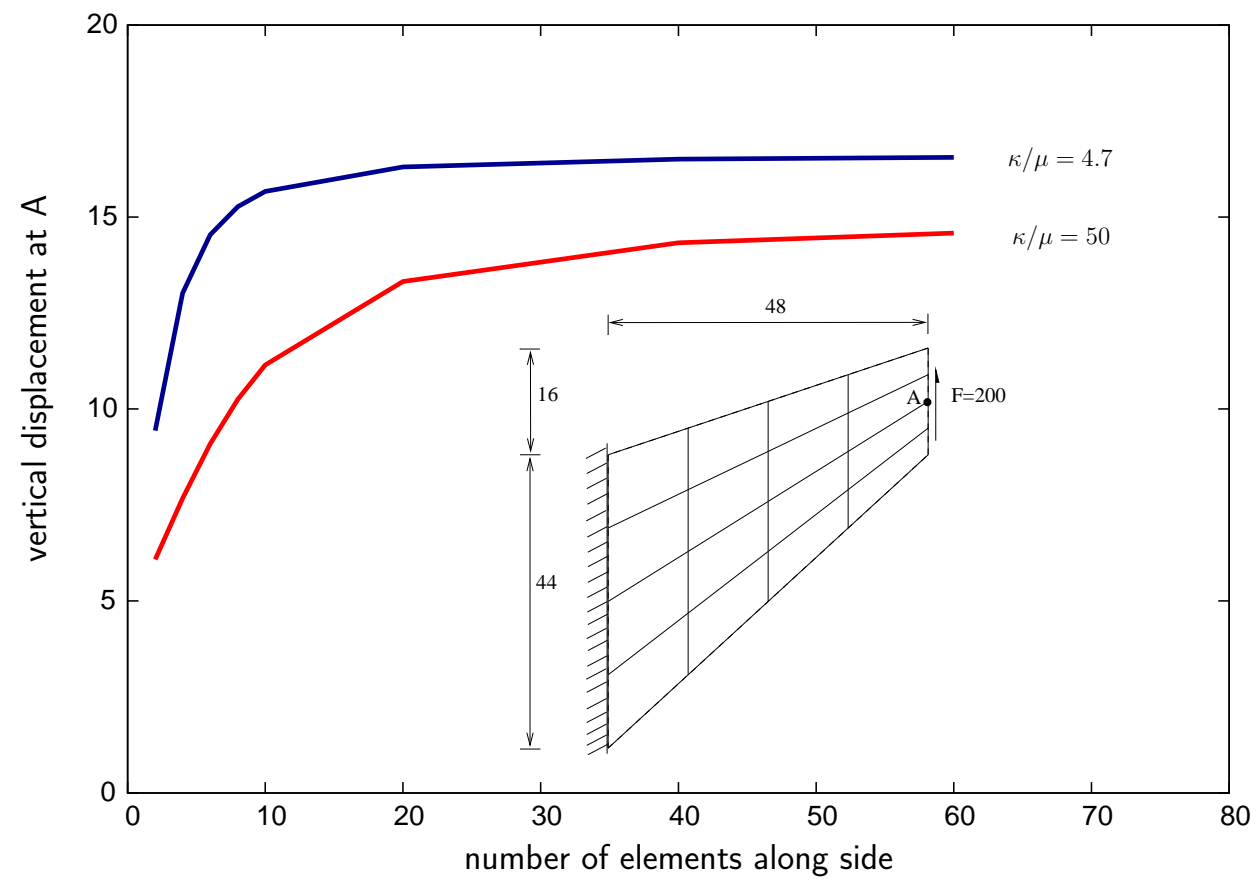

Figure 10: Cook's membrane: Problem geometry, boundary and loading conditions. Convergence behaviour for the compressible $(\kappa / \mu=4.7)$ and quasi-incompressible $(\kappa / \mu=50)$ polyconvex 2D-exponentiated Hencky model.

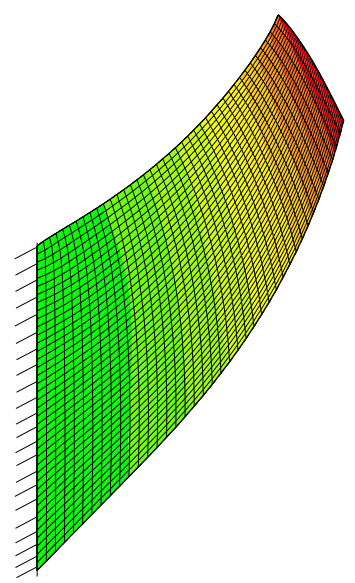

(a)

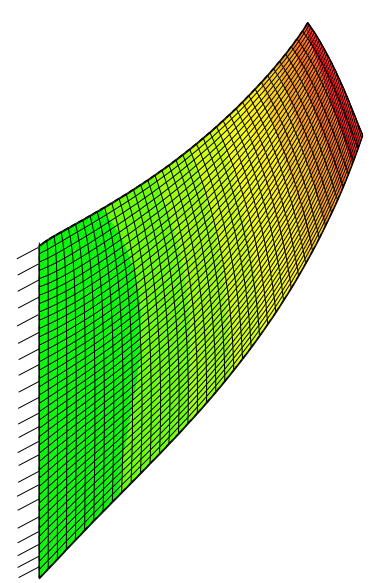

(b)

Figure 11: Cook's membrane for $40 \times 40$ mesh. Deformed configurations and vertical displacement fields: (a) with $\kappa / \mu=4.7$, and (b) with $\kappa / \mu=50$. 

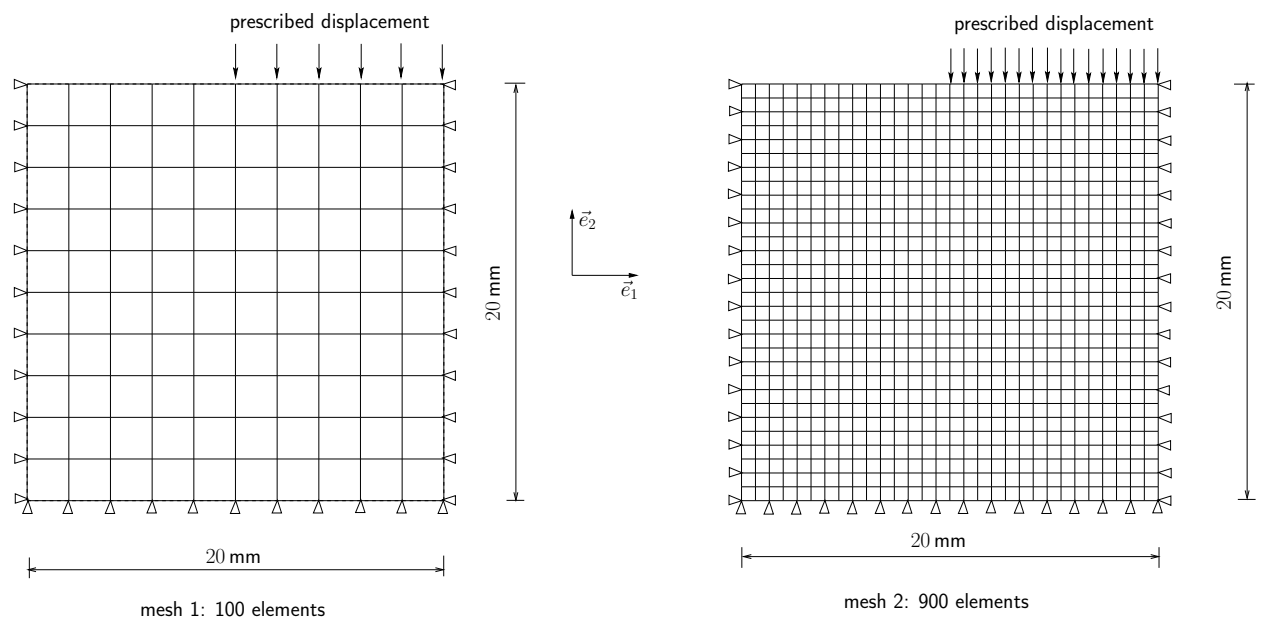

Figure 12: Planar footing example. Finite element meshes, boundary conditions and loading configuration.

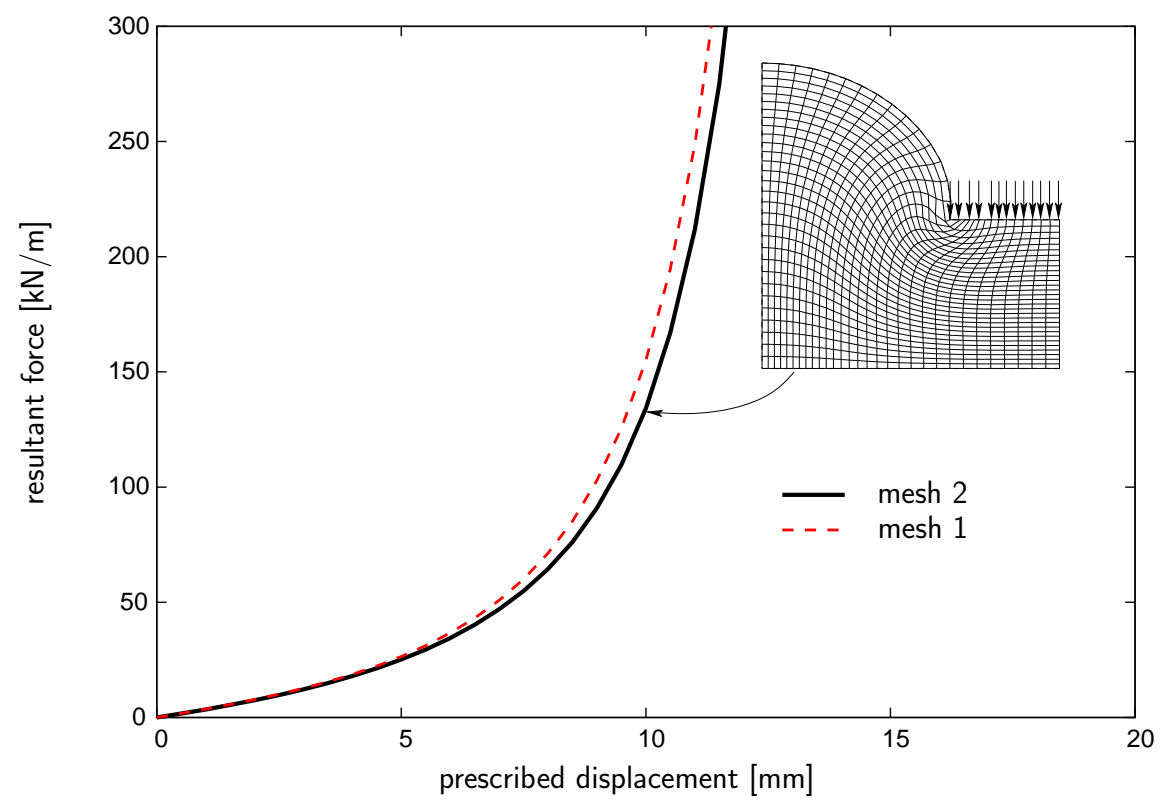

Figure 13: Resultant curves with the two meshes. Deformed configuration obtained with mesh 2 at prescribed displacement $\bar{v}_{\text {imp }}=-10 \mathrm{~mm}$. 


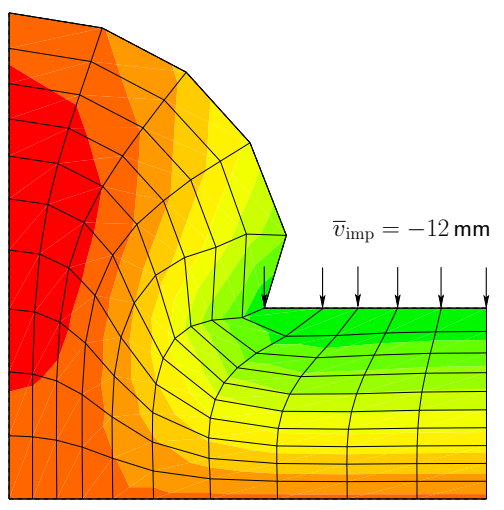

mesh 1

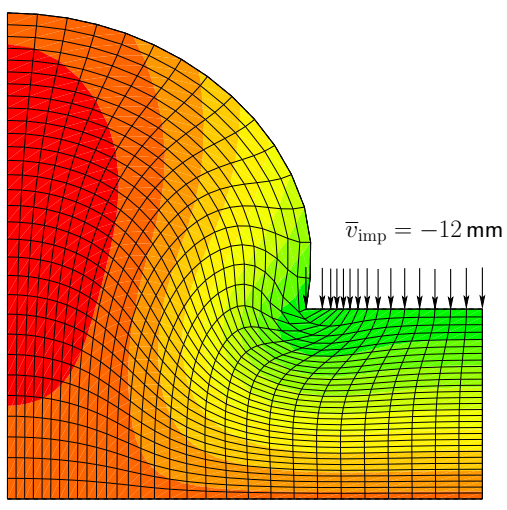

mesh 2

Figure 14: Deformed configurations and vertical displacement fields for the 2D-exponentiated Hencky model at $\bar{v}_{\text {imp }}=-12 \mathrm{~mm}$ for the two meshes.

\section{$7 \quad$ Eversion of a tube}

The so-called eversion of rubber tubes has been discussed as early as 1952 [9], when Gent and Rivlin investigated rubber tubes which are turned inside out experimentally and compared the findings with theoretical results in the large strain regime for incompressibility and isotropy. They obtained quite good agreements for $\partial W / \partial I_{1}$ and $\partial W / \partial I_{2}$ dependencies based on the Mooney-Rivlin type stored-energy function $W=c_{1}\left(I_{1}-3\right)+c_{2}\left(I_{2}-3\right)$, where $I_{1}, I_{2}$ are the first two deformation invariants. For Truesdell, the eversion of tubes was one of the most intriguing problems of nonlinear elasticity [46], cf. footnote 5. Many theoretical works can be found which try to provide analytic formulas. However, none of these approaches correctly describe the bulging at the upper and lower mantle. Indeed, in order to make the problem somehow tractable, the pointwise stress free condition at the upper and lower mantle is relaxed into a zero resultant stress condition. Closed form representations of the true eversion problem for unconstrained materials only exists for very unusual strain energies [5], which are, however, not useful to us ${ }^{8}$

Recently, the eversion problem has been considered by Liang et al. 20, who used equivalent experimental settings and provided descriptive photographs of inverted rubber tubes, see Figure 17.

Since the everted configuration satisfies equilibrium in the sense that

$$
\begin{aligned}
& \operatorname{div}_{\boldsymbol{X}} \boldsymbol{S}_{1}\left(\nabla_{\boldsymbol{X}} \varphi(\boldsymbol{X})\right)=0 \quad \text { for all } \boldsymbol{X} \in \mathcal{B}_{0} \\
& \text { and } \quad S_{1}\left(\nabla_{\boldsymbol{X}} \varphi(\boldsymbol{Y})\right) \boldsymbol{n}(\boldsymbol{X})=\mathbf{0} \quad \text { for all } \boldsymbol{X} \in \partial \mathcal{B}_{0} \text { with normal vector } \boldsymbol{n}(\boldsymbol{X}) \text {, }
\end{aligned}
$$

the eversion is a classic example of non-uniqueness of solutions to the traction problem in nonlinear elasticity [49.

For the eversion problem, we will only consider an incompressible material response.

${ }^{8}$ The compressible hyperelastic models considered in [5] require the elastic energy potential $W$ to be of the Valanis-Landel form 47

$$
W(F)=\sum_{i=1}^{3} w\left(\lambda_{i}\right)
$$

with a function $w:[0, \infty) \rightarrow \mathbb{R}$. In the past, energy functions of this type have been successfully applied in the incompressible case [47] 19], where they are in good agreement with experimental results. However, in the compressible case, an energy function of the form 66 without an additional volumetric energy term always implies zero lateral contraction for uniaxial stresses. 


\subsection{Implementation}

The implementation within the FE system ABAQUS is realized by the umat user-subroutine in order to obtain the Cauchy stress tensor $\boldsymbol{\sigma}=\boldsymbol{\tau} / \operatorname{det} \boldsymbol{F}$ as given in [15].

The representation of the spatial tangent operator $\widetilde{\boldsymbol{C}}$ in $\sqrt{33}$ is modified as discussed in [50] or more recently in [17. In that case, the bracketed term in 33 can be written as

$$
[\cdot]=\lambda_{k}^{2} \lambda_{l} \frac{\partial S_{2}^{k}}{\partial \lambda_{l}}
$$

i.e. as a function of the second Piola-Kirchhoff stress tensor $\boldsymbol{S}_{2}$ in the principal axis with $k, l=1,2,3$.

Afterwards, the resulting modulus $\widetilde{\boldsymbol{C}}$ is modified in each $i j k l$-term (with $i, j, k, l=1,2,3$ ) by

$$
\left\{\widetilde{\boldsymbol{C}}^{\mathrm{ABAQUS}}\right\}_{i j k l}=\{\widetilde{\boldsymbol{C}}\}_{i j k l}+\frac{1}{2}\left(\tau_{i k} \delta_{j l}+\tau_{j k} \delta_{i l}+\tau_{i l} \delta_{j k}+\tau_{j l} \delta_{i k}\right)
$$

in order to represent the Jaumann derivatives as expected by the ABAQUS environment for consistent linearization therein; here, again, $\delta_{a b}$ indicates the Kronecker symbol for $a, b=1,2,3$. Note that the ABAQUS implementation is fully hyperelastic with the correct linearization only in the incompressible case (in which the Cauchy stress $\sigma$ coincides with the Kirchhoff stress $\tau$ ). In the compressible case, the occurring error in the linearization can be overcome by modifying formula $(68)$, see [3, 4, 17] $9^{9}$

\subsection{Parameter fitting}

In order to realize a suitable parameter fit, we formulate $\sqrt{37}$ as

$$
\widetilde{S}_{1}^{i}=2 \mu \exp \left(k\left\{\ln ^{2} \lambda_{1}+\ln ^{2} \lambda_{2}+\ln ^{2} \lambda_{3}\right\}\right) \frac{\ln \lambda_{i}}{\lambda_{i}}
$$

in principal axis for (ideal) incompressibility with $\operatorname{det} \boldsymbol{F}=\lambda_{1} \lambda_{2} \lambda_{3}=J \equiv 1$ and $\lambda_{i}=J^{-1 / 3} \lambda_{i}=\lambda_{i}$.

By (69), the stress state is determined except for the hydrostatic pressure $p$, so the principal first PiolaKirchhoff stresses are given by

$$
S_{1}^{i}=-\frac{1}{\lambda_{i}} p+\widetilde{S}_{1}^{i}
$$

for $i=1,2,3$.

For uniaxial test data with deformation state $\boldsymbol{F}=\operatorname{diag}\left\{\lambda_{1}, \frac{1}{\sqrt{\lambda_{1}}}, \frac{1}{\sqrt{\lambda_{1}}}\right\}$ from the uniaxial stretch $\lambda_{1}$ and the stress boundary conditions $S_{1}^{2}=S_{1}^{3} \equiv 0$ in perpendicular direction, we obtain

$$
S_{1}^{1}=3 \mu \exp \left(\frac{3}{2} k \ln ^{2} \lambda_{1}\right) \frac{\ln \lambda_{1}}{\lambda_{1}}
$$

after some calculations from (69).

For a silicone rubber as given in Fig. 15, we obtain by a simple least square fit the free model parameters $\mu=G=0.612 \mathrm{MPa}$ and $k=1.173$. In comparison, the equivalent uniaxial stress-stretch result for a Neo-Hooke model using the above calibrated $\mu=G$ is given.

\footnotetext{
${ }^{9}$ In the (unmodified) compressible case, the ABAQus updated Lagrangian implementation is not energy consistent, since the used Jaumann-rate of the Cauchy stress

$$
\stackrel{\Delta}{\boldsymbol{\sigma}}=\dot{\boldsymbol{\sigma}}+\boldsymbol{\sigma} \cdot \boldsymbol{W}-\boldsymbol{W} \cdot \boldsymbol{\sigma}
$$

with $\boldsymbol{W}=\operatorname{skew}(\boldsymbol{L})=\frac{1}{2}\left(\boldsymbol{L}-\boldsymbol{L}^{T}\right)$ is not energy consistent with the Cauchy stress. The principle of virtual work must be implemented correctly for any choice of stress and objective stress-rate.
} 


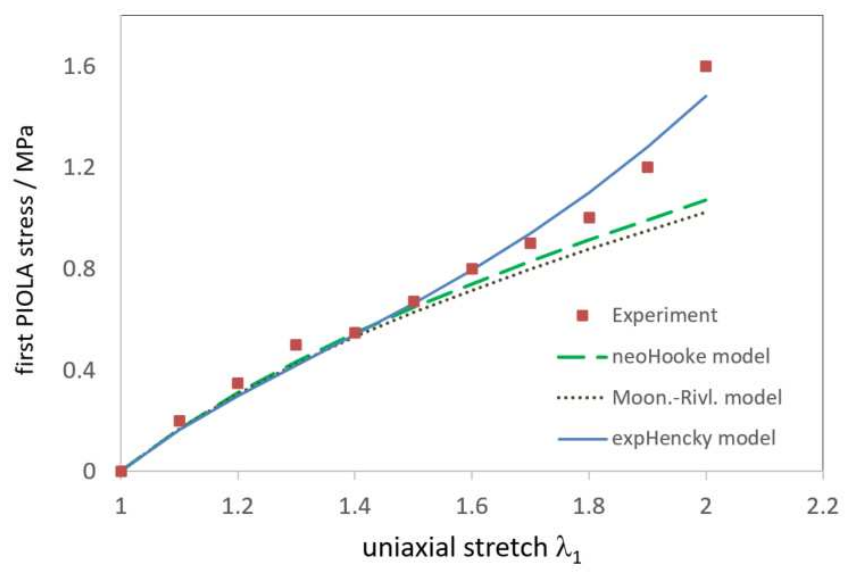

Figure 15: Uniaxial parameter fit for the exponentiated Hencky model; in comparison resulting Neo-Hooke model and Mooney-Rivlin model with $c_{1}=\frac{5}{11} G$ and $c_{2}=\frac{5}{110} G$, so that $c_{2}=\frac{1}{10} c_{1}$ and $G=2\left(c_{1}+c_{2}\right)$.

\subsection{Simulation and results}

We realize the numerical simulation of model experiments within the Finite Element Method as depicted in Fig. 18. Here, tubes with different inner radius $r$ are everted inside-out. The resulting deformation state with focus on the inner and the outer radius and on the axial length at the end of the process is observed for hyperelastic, time-independent material behaviour in the model. The eversion of the modeled tubes is realized by a given displacement of the double (axial) tube length at the outer circle signed in Fig. 18 in axial $z$-direction, whereas the nodes on the inner circle are fixed. Due to symmetry, just a quarter of the tube is modeled - with symmetry conditions at both cutting planes at $x \equiv 0$ and $y \equiv 0$. As a result, four different

\begin{tabular}{|c|c|c|c|c|}
\hline$\mu$ & $k$ & $L_{a x}$ & $r$ & $R$ \\
\hline $0.612 \mathrm{MPa}$ & 1.173 & $10 \mathrm{~mm}$ & $4.5 \mathrm{~mm}$ & $6.0 \mathrm{~mm}$ \\
\hline
\end{tabular}

Table 3: Geometry and material parameters.

deformation states at 20\%,50\%,75\% and $100 \%$ of eversion are shown in Fig. 19 here, the shaded contours represent the maximal principal logarithmic strain within the bulk.

In order to compare different material models, we show in Fig. 16 the (global) reaction force everting the tube models: All three models (Neo-Hooke, Mooney-Rivlin and exponentiated Hencky) are applied with comparable infinitesimal shear modulus $(\mu=G=0.612 \mathrm{MPa})$ as mentioned in Sect. 7.2 previously. Fig. 16 shows the overall (axial) reaction force vs. the ratio of eversion of the tube models. The applied models result in a typical course of compressing the tube in axial direction to more than half of axial deformation, and then turning the sign into a tension characteristics. Here, the exponentiated Hencky model shows this characteristics much earlier than the Mooney-Rivlin model, whereas the Neo-Hooke-type model seems to run through an instability point in that configuration of $r=4.5 \mathrm{~mm}$ and $R=6.0 \mathrm{~mm}$.

Further investigations might include varying the inner and the outer radius using different material models with comparable material parameters.

\section{Conclusion and perspectives}

In this paper, the variational setting of nonlinear elasticity based on the exponentiated Hencky model in finite strain elasticity has been investigated for an appropriate discretization in terms of the finite element 
method. The key approach in the design of an integration algorithm was a systematic use of the spectral decomposition of the stress and strain quantities. Among others, the common difficulties related to equal eigenvalues have been circumvented by use of the limits applying l'Hôspital rule.

We have presented complete details of the final expressions for an easy implementation within the context of the finite element method and, as shown, the set of numerical simulations has highlighted some pertinent features that demonstrate the efficiency and robustness of the proposed numerical formulation for threedimensional problems as well as for the particular planar exponentiated Hencky model. Finally, we have used the ABAQUS-FEM-procedure to simulate the eversion of an incompressible elastic tube, further demonstrating the overall usefulness of the three-dimensional exponentiated Hencky model. In the near future, we will adapt the ABAQUS framework to compressible nonlinear responses, which requires changes to the stiffness tensor as described by Bažant [3, 4, 17].

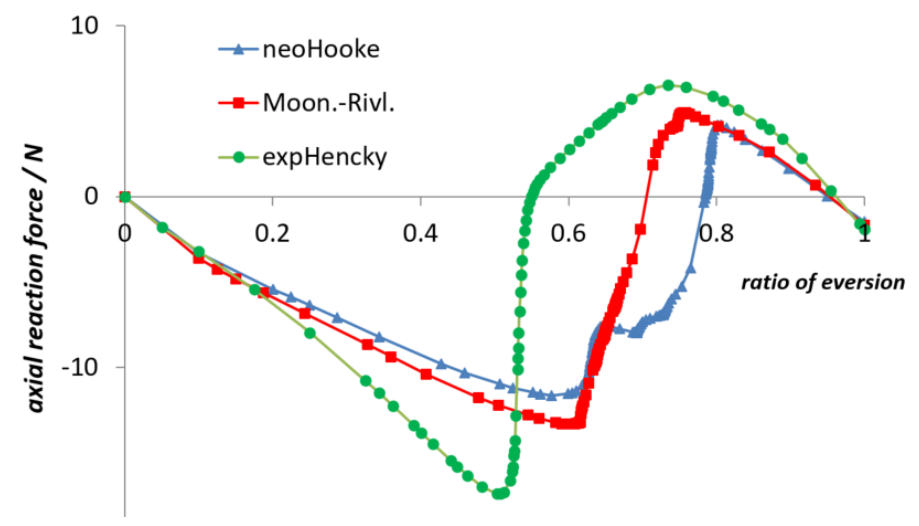

Figure 16: Global reaction force to evert the tubes. (Blue) triangles - Neo-Hooke; (red) squares - MooneyRivlin; (green) dots - exponentiated Hencky model
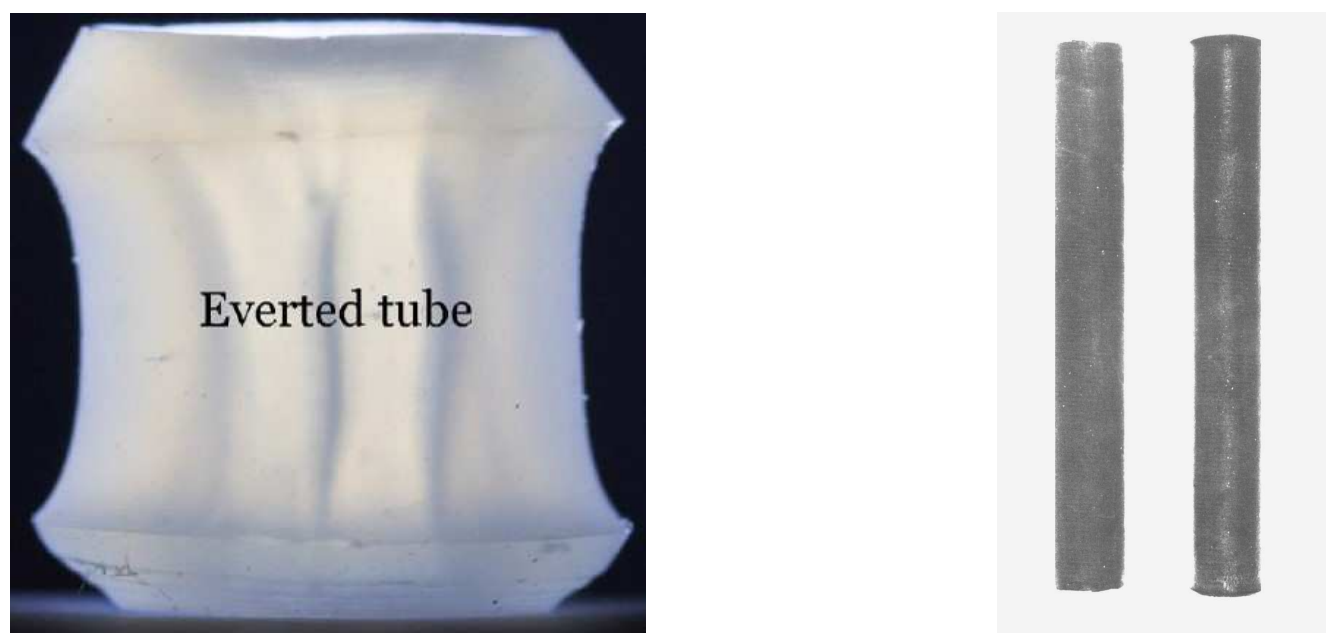

Figure 17: Left: Photo of an everted tube [20]. Right: Photo of rubber tubing before and after eversion [46]. 

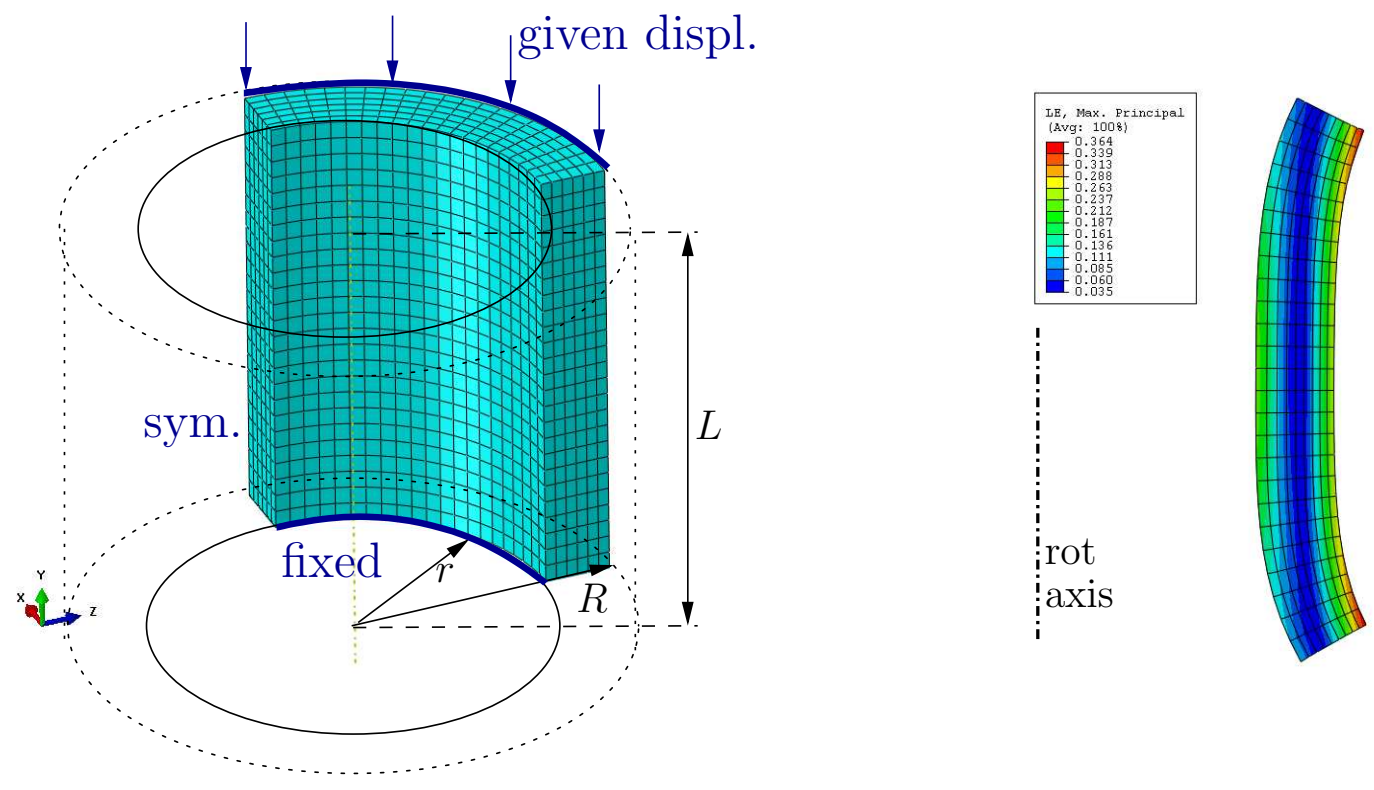

Figure 18: Left: Model of an elastomeric tube to be everted; variation of inner radius $r$. Right: Cross section of the fully everted elastic tube; note that apart from the flaring ends, the everted configuration still closely resembles a circular cylinder.
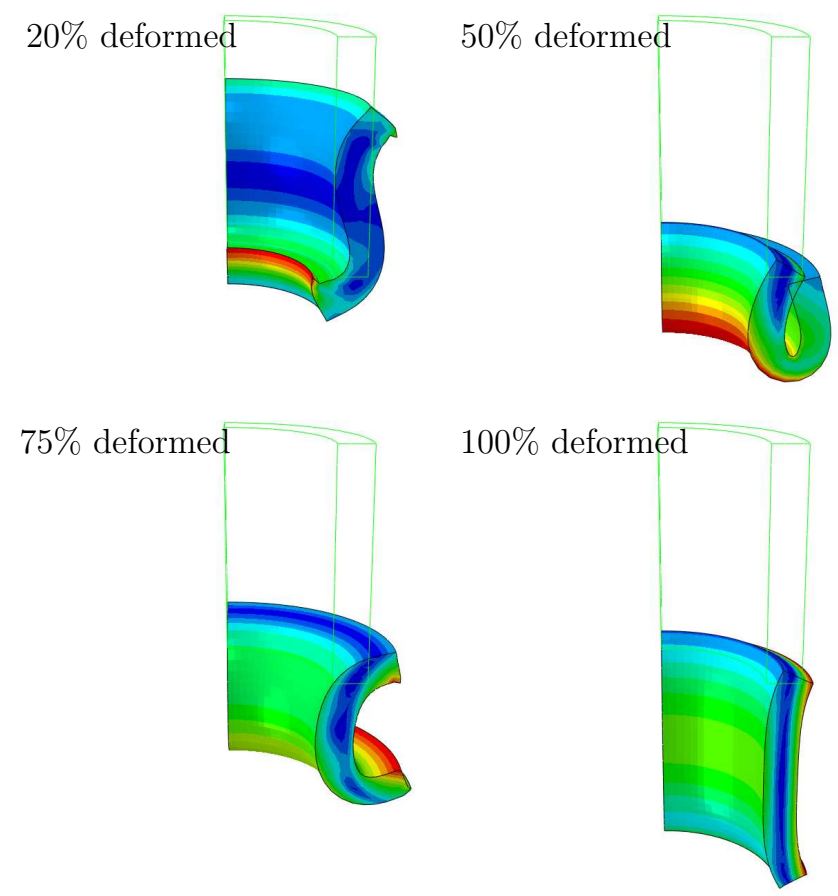

Figure 19: Eversion of an elastic tube: states of deformation. The maximum principal logarithmic strains occur at the places marked in red. 


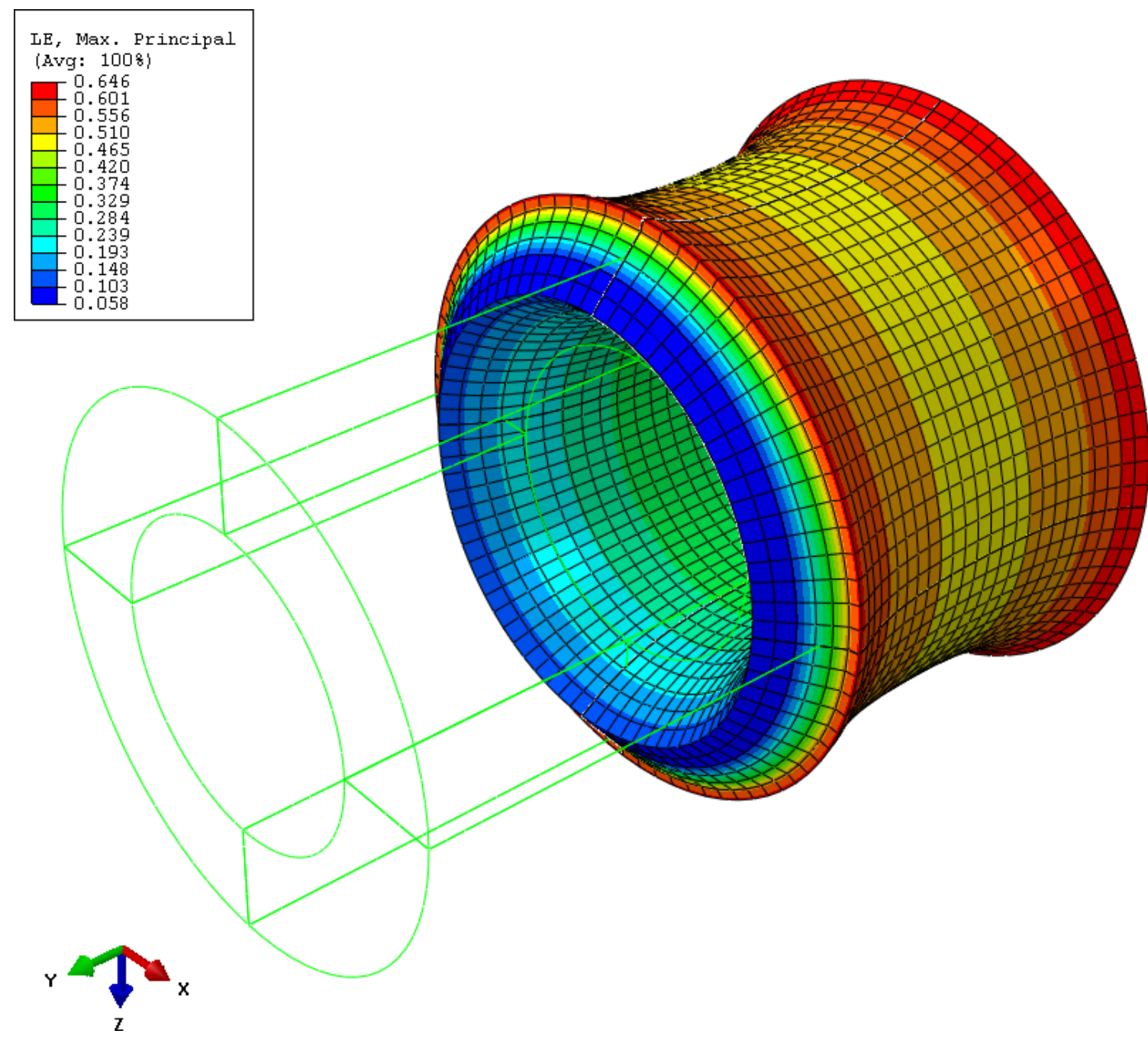

Figure 20: The fully everted elastic tube, with maximum occurring principal stretches in the order of about $200 \%$.

\section{References}

[1] L. Anand. On H. Hencky's approximate strain energy function for moderate deformations. Journal of Applied Mechanics, 46:78-82, 1979.

[2] F. Armero. Elastoplastic and viscoplastic deformations in solids and structures. In E. Stein, R. de Borst, and T. Hughes, editors, Encyclopedia of Computational Mechanics, vol. 2: Solids and Structures, pages 227-266. John Wiley \& Sons., 2004.

[3] Z. P. Bažant and J. Vorel. Objective stress rates in finite strain of inelastic solid and their energy consistency. Technical report, McCormick School of Engineering and Applied Science, Northwestern University, 2012.

[4] Z. P. Bažant, M. Gattu, and J. Vorel. Work conjugacy error in commercial finite-element codes: its magnitude and how to compensate for it. Proceedings of the Royal Society of London A: Mathematical, Physical and Engineering Sciences, 2012. 
[5] Y.-C. Chen and D. Haughton. Existence of exact solutions for the eversion of elastic cylinders. Journal of Elasticity, 49(1):79-88, 1997.

[6] W. Ehlers and G. Eipper. The simple tension problem at large volumetric strains computed from finite hyperelastic material laws. Acta Mechanica, 130(1):17-27, 1998.

[7] P. J. Flory. Thermodynamic relations for high elastic materials. Transactions of the Faraday Society, 57:829-838, 1961.

[8] A. N. Gent. A new constitutive relation for rubber. Rubber Chemistry and Technology, 69:59-61, 1996.

[9] A. N. Gent and R. S. Rivlin. Experiments on the mechanics of rubber I: eversion of a tube. Proceedings of the Physical Society B, 65:118-121, 1952.

[10] I.-D. Ghiba, P. Neff, and R. J. Martin. An ellipticity domain for the distortional Hencky logarithmic strain energy. Proceedings of the Royal Society of London. A. Mathematical and Physical Sciences, 471(2184), 2015.

[11] I.-D. Ghiba, P. Neff, and M. Šilhavỳ. The exponentiated Hencky-logarithmic strain energy. Improvement of planar polyconvexity. International Journal of Non-Linear Mechanics, 71:48-51, 2015.

[12] H. Hencky. Über die Form des Elastizitätsgesetzes bei ideal elastischen Stoffen. Zeitschrift für technische Physik, 9:215-220, 1928. available at wWw.uni-due.de/imperia/md/content/mathematik/ag_neff/ hencky1928.pdf.

[13] H. Hencky. Welche Umstände bedingen die Verfestigung bei der bildsamen Verformung von festen isotropen Körpern? Zeitschrift für Physik, 55:145-155, 1929. available at www.uni-due.de/imperia/ md/content/mathematik/ag_neff/hencky1929.pdf.

[14] H. Hencky. The elastic behavior of vulcanized rubber. Rubber Chemistry and Technology, 6(2):217-224, 1933. available at https://www.uni-due.de/imperia/md/content/mathematik/ag_neff/hencky_ vulcanized_rubber.pdf

[15] G. A. Holzapfel. Nonlinear Solid Mechanics. A Continuum Approach for Engineering. John Wiley and Sons, Ltd, Chichester, West Sussex, UK, 2000.

[16] T. J. R. Hughes. The Finite Element Method. Prentice-Hall, Englewood-Cliffs, NJ, 1987.

[17] W. Ji, A. M. Waas, and Z. P. Bažant. On the importance of work-conjugacy and objective stress rates in finite deformation incremental finite element analysis. Journal of Applied Mechanics, 80(4):041024, 2013.

[18] C. S. Jog and K. D. Patil. Conditions for the onset of elastic and material instabilities in hyperelastic materials. Archive of Applied Mechanics, 83(5):661-684, 2013.

[19] D. F. Jones and L. R. G. Treloar. The properties of rubber in pure homogeneous strain. Journal of Physics D: Applied Physics, 8(11):1285, 1975.

[20] X. Liang, F. Tao, and S. Cai. Creasing of an everted elastomer tube. Soft Matter, 12(37):7726-7730, 2016.

[21] R. J. Martin and P. Neff. Minimal geodesics on GL( $n)$ for left-invariant, right-O $(n)$-invariant riemannian metrics. Journal of Geometric Mechanics, 8(3):323-357, 2016. available at arXiv:1409.7849.

[22] C. Miehe. Aspects of the formulation and finite element implementation of large strain isotropic elasticity. International Journal for Numerical Methods in Engineering, 37(12):1981-2004, 1994. 
[23] L. A. Mihai and P. Neff. Hyperelastic bodies under homogeneous Cauchy stress induced by nonhomogeneous finite deformations. International Journal of Non-Linear Mechanics, 89:93-100, 2017. available at arXiv:1608.05040.

[24] L. A. Mihai and P. Neff. Hyperelastic bodies under homogeneous Cauchy stress induced by threedimensional non-homogeneous deformations. to appear in Mathematics and Mechanics of Solids, 2017. available at arXiv:1611.01772.

[25] G. Montella, S. Govindjee, and P. Neff. The exponentiated Hencky strain energy in modeling tire derived material for moderately large deformations. Journal of Engineering Materials and Technology, 138(3):031008-1 - 031008-12, 2016.

[26] B. Nedjar. Frameworks for finite strain viscoelastic-plasticity based on multiplicative decompositions. Part II: Computational aspects. Computer Methods in Applied Mechanics and Engineering, 191:15631593, 2002.

[27] B. Nedjar. On a continuum thermodynamics formulation and computational aspects of finite growth in soft tissues. International Journal for Numerical Methods in Biomedical Engineering, 27:1850-1866, 2011.

[28] B. Nedjar. On constitutive models of finite elasticity with possible zero apparent Poisson's ratio. International Journal of Solids and Structures, 91:72-77, 2016.

[29] P. Neff. Mathematische Analyse multiplikativer Viskoplastizität. Ph.D. Thesis, Technische Universität Darmstadt. Shaker Verlag, Aachen, 2000. available at http://www.uni-due.de/ hm0014/Download_ files/neffdiss.ps.

[30] P. Neff, B. Eidel, and R. J. Martin. The axiomatic deduction of the quadratic Hencky strain energy by Heinrich Hencky. arXiv:1402.4027, 2014.

[31] P. Neff, B. Eidel, and R. J. Martin. Geometry of logarithmic strain measures in solid mechanics. Archive for Rational Mechanics and Analysis, 222(2):507-572, 2016. available at arXiv:1505.02203.

[32] P. Neff and I.-D. Ghiba. The exponentiated Hencky-logarithmic strain energy. Part III: Coupling with idealized isotropic finite strain plasticity. Continuum Mechanics and Thermodynamics, 28(1):477-487, 2016.

[33] P. Neff and I.-D. Ghiba. Loss of ellipticity for non-coaxial plastic deformations in additive logarithmic finite strain plasticity. International Journal of Non-Linear Mechanics, 81:122-128, 2016. available at arXiv:1410.2819.

[34] P. Neff, I.-D. Ghiba, and J. Lankeit. The exponentiated Hencky-logarithmic strain energy. Part I: Constitutive issues and rank-one convexity. Journal of Elasticity, 121(2):143-234, 2015.

[35] P. Neff, J. Lankeit, I.-D. Ghiba, R. J. Martin, and D. J. Steigmann. The exponentiated Henckylogarithmic strain energy. Part II: coercivity, planar polyconvexity and existence of minimizers. Zeitschrift für angewandte Mathematik und Physik, 66(4):1671-1693, 2015.

[36] P. Neff and L. A. Mihai. Injectivity of the Cauchy-stress tensor along rank-one connected lines under strict rank-one convexity condition. Journal of Elasticity, 127(2):309-315, 2017. available at arXiv:1608.05247.

[37] R. W. Ogden. Non-linear Elastic Deformations. Dover, New York, 1997.

[38] J. Plešek and A. Kruisová. Formulation, validation and numerical procedures for Hencky's elasticity model. Computers and Structures, 84:1141-1150, 2006. 
[39] S. Reese and S. Govindjee. A theory of finite viscoelasticity and numerical aspects. International Journal of Solids and Structures, 35(26-27):3455-3482, 1998.

[40] H. Richter. Das isotrope Elastizitätsgesetz. Zeitschrift für Angewandte Mathematik und Mechanik, 28(7/8):205-209, 1948. available at https://www.uni-due.de/imperia/md/content/mathematik/ag_ neff/richter_isotrop_log.pdf

[41] W. C. Röntgen. Über das Verhältniss der Quercontraction zur Längendilatation bei Kautschuk. Annalen der Physik, 235(12):601-616, 1876.

[42] J. Schröder, M. von Hoegen, and P. Neff. The exponentiated Hencky energy: Anisotropic extension and biomechanical applications. to appear in Computational Mechanics, 2017. available at arXiv:1702.00394.

[43] J. C. Simo. Numerical analysis and simulation of plasticity. In P. Ciarlet and J. Lions, editors, Handbook of Numerical Analysis, vol. VI, pages 183-499. North-Holland, 1998.

[44] J. C. Simo and T. J. R. Hughes. Computational Inelasticity. Springer-Verlag, New York, 1998.

[45] C. Truesdell. Mechanical foundations of elasticity and fluid dynamics. Journal of Rational Mechanics and Analysis, 1:125-300, 1952.

[46] C. Truesdell. Some challenges offered to analysis by rational thermomechanics: three lectures for the international symposium on continuum mechanics and partial differential equations. North-Holland Mathematics Studies, 30:495-603, 1978.

[47] K. C. Valanis and R. F. Landel. The strain-energy function of a hyperelastic material in terms of the extension ratios. Journal of Applied Physics, 38(7):2997-3002, 1967.

[48] C. Vallée. Lois de comportement élastique isotropes en grandes déformations. International Journal of Engineering Science, 16(7):451-457, 1978.

[49] J. L. Wegner and J. B. Haddow. Elements of Continuum Mechanics and Thermodynamics. Cambridge University Press, 2009.

[50] P. Wriggers. Nonlinear Finite Element Methods. Springer-Verlag, Berlin, Heidelberg, 2008.

[51] O. C. Zienkiewicz and R. L. Taylor. The Finite Element Method, 5th Ed., Volume 1. ButterworthHeinemann, Oxford, UK, 2000. 